\title{
belarus-
}

analysen

http://www.laender-analysen.de/belarus/

\section{BELARUS UND DIE UKRAINE-KRISE}

ANALYSE

Wachsende Angst vor Souveränitätsverlust

Die belarussische Außenpolitik im Zeichen des Ukraine-Konflikts

Andrej Fjodarau, Minsk

- UMFRAGE

$\begin{array}{lr}\text { Umfragen zur Ukraine-Krise } & 6\end{array}$

ANALYSE

Unter Schock: Die belarussische Wirtschaft im Kontext des Ukraine-Konflikts

Robert Kirchner, Berlin

- STATISTIK

BIP, Wechselkurs, Im- und Export

DOKUMENTATION

Zur Lage ukrainischer Flüchtlinge in Belarus

CHRONIK

1. Oktober bis 10. Dezember 2014 


\title{
Wachsende Angst vor Souveränitätsverlust
}

\section{Die belarussische Außenpolitik im Zeichen des Ukraine-Konflikts}

\author{
Andrej Fjodarau, Minsk
}

\section{Zusammenfassung:}

Russlands aggressive Politik gegenüber der Ukraine hat bei der belarussischen Führung erkennbare Ängste ausgelöst, dass der Kreml mittelfristig auch die Souveränität von Belarus bedrohen könnte. Um dieser Gefahr zu begegnen, müsste das offizielle Minsk durch ernsthafte Reformen die Voraussetzungen für eine substantielle Verbesserung der Beziehungen zum Westen zu schaffen. Bisher beschränken sich die belarussischen Bemühungen jedoch auf die rhetorische Unterstützung für die neue ukrainische Führung im Konflikt mit Russland. Dies hat sich vorerst als nicht ausreichend für die erhoffte Normalisierung der Beziehungen zur EU erwiesen.

$\mathrm{D}$ ie russische Aggression gegen die Ukraine hat die bisher in Europa geltende Ordnung, der zufolge keine gewaltsame Aneignung von Gebieten anderer Staaten erfolgt, in Frage gestellt. Mit seinem Vorgehen hat Moskau unmissverständlich demonstriert, dass es nicht die Absicht hat, sich an geltende internationale Vereinbarungen zu halten, und diese nach eigener Willkür interpretiert. Dementsprechend alarmiert reagierte die internationale Staatengemeinschaft. Besonders hoch ist die Besorgnis verständlicherweise bei den in Russlands Nähe gelegenen Staaten. Ungeachtet der besonderen Beziehungen, die das offizielle Minsk mit Moskau verbinden, stellt Belarus in dieser Hinsicht keine Ausnahme dar. Für das Land hat sich vielmehr die ohnehin nicht einfache außenpolitische Lage, in der es sich in den letzten Jahren befand, nochmals erheblich verkompliziert.

\section{Rhetorische Eigenständigkeit}

Aus den diesjährigen Reden Aljaksandr Lukaschenkas lässt sich deutlich erkennen, dass ihm die um die Ukraine entstandene Situation Unbehagen bereitet. Bisweilen konnte sogar der Eindruck entstehen, dass er eine klare Gegenposition zum Kreml einnimmt: So hielt Lukaschenka, als Putin die neue Kiewer Führung ignorierte, aktiv den Kontakt; als Moskau die Föderalisierung der Ukraine verlangte, trat Minsk für ihre territoriale Integrität ein und als der Kreml mit »Freiwilligen" und Waffen die Separatisten unterstützte, rief der belarussische Präsident dazu auf, diese zu bekämpfen. Allerdings beschränkte sich die Eigenständigkeit der belarussischen Politik lediglich auf die Rhetorik. In der Praxis verhielt sich das offizielle Minsk hingegen durchgängig loyal zur Politik des Kremls. Besonders deutlich zeigte sich dies daran, dass Belarus in der UN-Generalversammlung gegen die Resolution über die territoriale Integrität der Ukraine stimmte und die Stationierung zusätzlicher russischer Luftstreitkräfte auf belarussischem Gebiet erlaubte.
In der Vergangenheit ist es der belarussischen Führung freilich durchaus gelungen, Russland bei seiner Politik im postsowjetischen Raum die blinde Gefolgschaft zu verweigern. Insbesondere erkannte Belarus nicht die Unabhängigkeit von Abchasien und Südossetien an. Dieses Mal ist die Loyalität des engsten Bündnispartners für den Kreml jedoch wesentlich wichtiger. Zudem hat Belarus inzwischen deutlich weniger Widerstandsmöglichkeiten. Denn infolge der schwierigen wirtschaftlichen Lage sowie der verschlechterten Beziehungen zum Westen hat sich die Abhängigkeit von Moskau in den letzten Jahren erheblich erhöht. Deswegen sind die Bemühungen der belarussischen Führung, mit ihren Äußerungen zur Ukraine-Krise sowie durch ihre Kontakte zur Kiewer Führung eine unabhängige Position zu demonstrieren, nicht mehr als der Versuch, eine gute Miene zum bösen Spiel zu bewahren.

\section{Angst vor Inkorporation}

Gleichzeitig ist die wachsende Angst des offiziellen Minsk vor einer drohenden Inkorporation durch Russland unverkennbar. Während der letzten Monate hat Lukaschenka mehrmals seine Besorgnis geäußert, dass das ukrainische Szenario sich in Belarus wiederholen könnte. Bei seiner Jahresansprache vor der Nationalversammlung am 22. April betonte er die Bereitschaft, die staatliche Souveränität und Unabhängigkeit mit allen verfügbaren Mitteln zu verteidigen. Monate später wurde er noch deutlicher, indem er davon sprach, dass die prorussischen Terroristen und Kämpfer im Falle eines Misserfolgs in der Ostukraine sich Belarus vornehmen könnten. Und am 17. Oktober erklärte Lukaschenka gegenüber russischen Lokaljournalisten, dass Russland imperiale Ambitionen verfolge. Zugleich verurteilte er scharf alle Anschuldigungen, dass in Belarus die russischsprachige Bevölkerung diskriminiert werde, und beauftragte die Sicherheitsorgane, entsprechende Äußerungen umgehend zu unterbinden. Wie zurückhaltend die belarussische Führung panslawis- 
tische Bewegungen im eigenen Land betrachtet, zeigt sich auch daran, dass dem Belarussischen Slawischen Komitee seit 2011 die Umwandlung in eine politische Partei verweigert wird.

Diese Maßnahmen bieten jedoch keine Garantie, dass die Gefahr gebannt werden kann. Denn es wäre naiv zu glauben, dass eine russische Aggression gegenüber Belarus genau nach dem ukrainischen Szenario erfolgen wird, d. h. unter dem Vorwand, die Interessen der russischsprachigen Bevölkerung zu verteidigen. Sollte Moskau dieses Ziel verfolgen, kann es für eine Aggression jeden beliebigen Vorwand nutzen - bis hin zum Vorwurf des in Belarus bestehenden Demokratiedefizits. Die von Lukaschenka geäußerte These, dass die Russen im Falle einer Putinschen Aggression auf der Seite der Belarussen kämpfen würden, kann nicht überzeugen. Vielmehr gibt es ausreichend Gründe das Gegenteil anzunehmen, nämlich dass die von Lukaschenka so gepriesenen belarussischen Streitkräfte nicht einmal versuchen würden, einem Einmarsch aus dem Osten Widerstand zu leisten. Für diese Annahme spricht, dass viele Belarussen in Russland keinen Aggressor sehen und es in den staatlichen Strukturen zahlreiche verdeckte Agenten gibt. Hinzu kommen der enorme Einfluss der russischen Fernsehsender sowie die Bereitschaft zahlreicher "asozialer Elemente" für ein geringes Entgelt die Rolle der hiesigen »Tituschki«, wie die Schlägertruppen des ehemaligen Präsidenten Viktor Janukowitsch in der Ukraine genannt werden, zu übernehmen.

\section{Konfliktbehaftete Integration}

Das zentrale Instrument, mit der Wladimir Putin derzeit seine geopolitischen Ziele verfolgt, ist die Eurasische Wirtschaftsunion (EAWU). Zwar bestreitet der russische Präsident öffentlich, dass er die Absicht verfolge, mit Hilfe der EAWU die UdSSR wiederherzustellen. Gleichzeitig strebt der Kreml jedoch erkennbar danach, der EAWU einen supranationalen Charakter zu geben. Dies zeigt sich beispielsweise an dem hartnäckigen Bemühen, in den Gründungvertrag Absprachen über konkrete Bausteine für einen politischen Überbau zu integrieren, wie über die Bildung einer interparlamentarischen Versammlung, die Koordination der Außenpolitik sowie die Kooperation im Außenhandel.

Bis jetzt ist es Belarus und Kasachstan gelungen, diese Initiativen zurückzuweisen. Doch Moskau verfügt über viele Möglichkeiten, um seine »Partner« zum Einlenken zu zwingen. Das offizielle Minsk sollte sich daher nicht zu sehr darüber freuen, dass es ihm im Vorfeld der Ratifizierung des EAWU-Vertrags gelungen ist, Zugeständnisse im Erdölsektor zu erreichen. Denn es gibt keinerlei Garantien dafür, dass die alten Widersprüche nach dem Inkrafttreten des Vertrags nicht wieder an die Oberfläche treten, und zwar in noch deutlicher Form. Hierfür sprechen folgende Annahmen:

1. Im Zuge des fortschreitenden Integrationsprozesses der EAWU wird sich Belarus einem Ansturm finanzstarker russischer Wirtschaftskreise gegenübersehen, die sich die besten einheimischen Unternehmen aneignen möchten.

2. Sobald Moskau die Vormachtstellung in der Wirtschaft übernommen hat, kann die russische Führung auch die belarussische Außen- und Innenpolitik praktisch vollständig bestimmen. Da der Westen die EAWU als ein antieuropäisches Projekt wahrnimmt, kann Belarus zudem jegliche Annäherung an die EU vergessen.

3. Eine echte Wirtschaftsunion impliziert die Delegation beträchtlicher Vollmachten an die supranationale Ebene. Autoritäre Herrscher verzichten jedoch in der Regel ungern auf den Alleinherrschaftsanspruch in ihren Ländern. Die Wirtschaft stellt hier keine Ausnahme dar.

Aus diesen Gründen sind neue heftige Konflikte zwischen den EAWU-Mitgliedern zu erwarten. Erste Anzeichen hierfür gibt es bereits. Am 11. November verlangte Lukaschenka öffentlich, die einheimischen Produzenten mit allen Mitteln zu verteidigen, auch entgegen den Integrationsabsprachen. Wenig später stoppte Russland fast den gesamten Import von Lebensmitteln aus Belarus. Es ist offensichtlich, dass eine Wirtschaftsunion unter derartigen Rahmenbedingungen nicht funktionieren kann. Sollten die bestehenden Widersprüche weiter zunehmen und einen kritischen Punkt überschreiten, wird die EAWU das gleiche Schicksal erleiden wie alle anderen bisher von Russland initiierten Integrationsgebilde. In diesem Falle steigt die Wahrscheinlichkeit, dass Russland auch gegenüber Belarus auf gewaltsame Szenarien zurückgreift.

\section{Alternative Partner}

Angesichts dieser wachsenden Gefahr ist Belarus zunehmend auf äußere Unterstützung angewiesen. Es wäre falsch zu sagen, dass die belarussische Führung diesen Bedarf nicht sehen würde. In Übereinstimmung mit der deklarierten "multivektoralen« Außenpolitik versucht sie vielmehr bereits seit etlicher Zeit - wenn auch mit bescheidenen Erfolgen - die Zusammenarbeit mit anderen Staaten in Asien, Afrika und Lateinamerika zu entwickeln. Besondere Hoffnungen setzt Minsk in den »strategischen Partner» China. So erklärte Aljaksandr Lukaschenka beim Treffen mit dem ersten stellvertretenden chinesischen Premierminister Zhang Gaoli Ende September: "Die Unterstützung durch China ist ein Faktor für die Unabhängigkeit unseres Staates«. Das offizielle Minsk setzt also auf China als zentrale Stütze 
in seinem Widerstand gegen eine eventuelle russische Expansion. Allerdings ist diese Strategie wenig erfolgversprechend. Denn der Anteil Chinas am belarussischen Außenhandelsumsatz ist mit 4,2\% in 2013 und $3,8 \%$ im ersten Halbjahr 2014 weiterhin gering und weist zudem ein für Belarus negatives Saldo auf. Vor allem aber wird Peking sich niemals wegen Belarus auf eine Konfrontation mit Moskau einlassen.

Grundsätzlich wären die Länder des Westens der natürlichere Bündnispartner für Belarus. Das offizielle Minsk äußert durchaus Interesse an einer Normalisierung der Beziehungen. Tatsächlich lässt sich in jüngster Zeit eine Tendenz zur Abnahme der bestehenden Spannungen beobachten. Die Spitze des belarussischen Außenministeriums nimmt regelmäßig an unterschiedlichen Veranstaltungen teil, welche die EU im Rahmen der Initiative "Östliche Partnerschaft» (ÖP) durchführt. Ebenso wurden Verhandlungen über ein Visaerleichterungsabkommen aufgenommen und ein Dialog über Modernisierungsfragen gestartet. Auch die diplomatischen Kontakte mit den USA haben deutlich zugenommen. Beispielsweise fand am 22. September in New York das erste belarussisch-amerikanische Investitionsforum statt.

Die belarussische Führung versuchte darüber hinaus ihr Image dadurch zu verbessern, dass es sich als Vermittler im russisch-ukrainischen Konflikt anbot. Aber die bloße Bereitstellung von Minsk als Verhandlungsort hat Washington und Brüssel offensichtlich nicht besonders beeindruckt, wie sich an der erneuten Verlängerung der EU-Sanktionen Ende Oktober gezeigt hat. Damit bestätigten sich die Experteneinschätzungen nicht, die prognostiziert hatten, dass der Westen aufgrund der belarussischen Friedensbemühungen im Ukraine-Konflikt bereit sein würde, die Augen vor den weiterhin bestehenden Menschenrechtsproblemen zu schließen, um zu erreichen, dass sich Belarus in dieser Frage dauerhaft von Moskau distanziert. Wie sehr die belarussische Führung auf eine entsprechende Honorierung ihrer Ukraine-Politik gesetzt hatte, zeigte sich u. a. daran, dass das belarussische Außenministerium am 20. Oktober in kategorischer Form von den in Belarus akkreditierten EU-Botschaftern verlangte, die EU solle unverzüglich alle gegen das Land verhängten Sanktionen aufheben.

\section{Scheitern der ÖP}

In der Tat ist es nicht wünschenswert, dass die Isolation von Belarus durch den Westen verstärkt wird, da diese eindeutig dem Kreml in die Hände spielt. Der vielversprechendste Ansatz gegenüber dem Land besteht vielmehr darin, eine allmähliche Europäisierung des Landes durch die Ausweitung der Kontakte in den unterschied- lichsten Bereichen zu erreichen. Voraussetzung hierfür ist, dass der Westen tatsächlich ein ernsthaftes Interesse an einem unabhängigen und demokratischen Belarus hat. Insgesamt muss man heute leider feststellen, dass die Östliche Partnerschaft, die zu einer Annäherung der postsowjetischen Staaten an die EU führen sollte, keine sichtbaren Erfolge vorzuweisen hat. Hierfür lassen sich folgende Gründe nennen:

1. Im Unterschied zu den mitteleuropäischen und den baltischen Staaten hatten in den postsowjetischen Staaten weder die Mehrheit der Bevölkerung noch die politische Führung den brennenden Wunsch, Teil des vereinten Europas zu werden.

2. Innerhalb der Europäischen Union gab es diametral entgegengesetzte Vorstellungen über die letztendlichen Ziele der angestoßenen Partnerschaftsinitiative. Zudem erwiesen sich die Auswirkungen der Weltwirtschaftskrise als zusätzlicher Bremsfaktor.

3. Russland leistete aktiven Widerstand, da dem Kreml die möglichen Perspektiven der Partnerschaftsinitiative missfielen und zugleich seine Möglichkeiten, Druck auf die Nachbarn und die EU auszuüben, deutlich zunahmen.

Wenn der Kreml aus der gegenwärtigen Konfrontation zwischen Russland und dem Westen als Sieger hervorgehen sollte, werden alle Mitgliedsstaaten der Östlichen Partnerschaft früher oder später in den russischen Orbit hineingezogen werden. Im anderen Falle wird jedes Partnerland bilateral mit der EU sein Kooperationsniveau bestimmen. Und da sich die Interessen der Staaten in dieser Hinsicht kardinal unterscheiden, dürfte die Östliche Partnerschaftsinitiative ihre Existenzberechtigung verlieren. Belarus dürfte in beiden Fällen innerhalb der russischen Einflusszone bleiben - es sei denn den USA und der EU würde es gelingen, eine deutliche Abkühlung der Moskauer Herrschaftsambitionen zu erreichen. Bisher sieht es jedoch nicht danach aus. Es erscheint zudem fraglich, ob die von belarussischer Seite mehrmals geforderten Verhandlungen zwischen der EU und der Eurasischen Wirtschaftsunion zur Herstellung eines gemeinsamen Wirtschaftsraums diesem Ziel dienen können.

\section{Mögliche Szenarien}

Die dramatisch gestiegene Aggressivität des Kremls und seine Idee, die »russische Welt« ohne Rücksicht auf die Souveränität der postsowjetischen Staaten zu einigen, stellt Belarus vor ernsthafte Probleme. Die Politik, welche die heutige Führung in den letzten 20 Jahren verfolgt hat, hat das Land politisch und wirtschaftlich in hohem Maße von Russland abhängig gemacht. Der Kreml kann daher alle Versuche, sich seiner Position zu widersetzen, schnell und hart unterbinden. Man muss zugeben, dass 
die belarussische Führung bisher in der Ukraine recht erfolgreich einen Balanceakt ausübt. Dies ist jedoch lediglich als taktischer Erfolg zu bewerten. Die strategischen Perspektiven sind hingegen wenig erfreulich.

Falls sich Russland zu einem direkten militärischen Eingreifen in der Ukraine entscheiden sollte, würde Belarus unvermeidlich auf die eine oder andere Weise einbezogen werden - was für das Land eine Katastrophe wäre. Sollte Moskau von dieser Variante Abstand nehmen, so wird es doch weiterhin wirtschaftlichen und politischen Druck auf die Ukraine ausüben - und diesem Druck wird sich das offizielle Minsk auf lange Sicht anschließen müssen mit allen negativen Konsequenzen, die damit für das eigene Land insbesondere im wirtschaftlichen Bereich verbunden sind.

Wenn die patriotische Hysterie der russischen Bevölkerung langfristig anhält, könnte diese vom Kreml zudem zusätzliche Schritte zur Wiederherstellung des Imperiums verlangen. Angesichts fallender Umfragewerte und der sich zunehmend verschlechternden Wirtschaftslage könnte sich Putin daher vor diesem Hintergrund entscheiden, die Inkorporation von Belarus tatsächlich zu vollziehen. Bisher hatte der Kreml es nicht nötig, seine Ziele in Belarus mit Gewalt durchzusetzen. Falls Minsk sich jedoch ernsthaft widersetzen sollte, könnte ein gewaltsames Szenario ohne Zögern zur Anwendung kommen, da Russland nach der Annexion der Krim keine Rücksicht mehr auf sein Image nehmen muss.

\section{Fragile Souveränität}

Die belarussische Führung hat das Land in immer neue Integrationsprojekte mit Russland geführt. Die eurasische Integration unter Moskauer Führung birgt für Belarus die reale Gefahr, seine Souveränität zu verlieren, selbst wenn dies erst in fernerer Zukunft geschehen sollte. Die lebensnotwendige außenpolitische Unterstützung könnte nur im Falle einer maximalen Annäherung an die EU und die USA erzielt werden. Leider gibt es derzeit keine Grundlage für entsprechende Hoffnungen, da Minsk nicht die geringste Bereitschaft zur Veränderung seiner antidemokratischen Innenpolitik erkennen lässt. Alle in letzter Zeit geführten Verhandlungen zielen nicht auf ernsthafte Veränderungen, sondern diese tragen lediglich begrenzten Charakter. Dementsprechend bleibt nur die Hoffnung darauf, dass der Westen eine russische Annexion nicht anerkennen würde.

Insgesamt nähert sich für Belarus somit der Moment der Wahrheit. Die Souveränität des Landes erscheint derzeit sehr verletzbar zu sein. Gute Aussichten auf ihren Erhalt dürften nur dann bestehen, wenn Russland aufgrund einer akuten politischen und wirtschaftlichen Instabilität im eigenen Land die Ressourcen für die Umsetzung seiner imperialen Ambitionen ausgehen.

Übersetzung: Astrid Sahm

\section{Über den Autor:}

Dr. Andrej Fjodarau (geb. 1949) ist Experte für außen- und sicherheitspolitische Fragen in Minsk und arbeitet u. a. für die Belarussische Analytische Werkstatt (<www.belaw.eu $>$ ) in Warschau.

Lesetipps:

- Belarusian Institute for Strategic Studies: Battle for Ukraine: The fog of war has not dispersed yet, BISS Blitz, BB \#03/2014EN, 5. November 2014, <http://belinstitute.eu/sites/biss.newmediahost.info/files/attached-files/BISS_ Blitz03_2014en.pdf>.

- Arkady Moshes: Belarus' Renewed Subordination to Russia. Unconditional Surrender or Hard Bargain? Ponars Eurasia Policy Memo No. 329, August 2014, <http://www.ponarseurasia.org/sites/default/files/policy-memos-pdf/ Pepm329_Moshes_August2014_2.pdf>.

- Andrej Jegorow: "Jesli Rossija ustanowit control nad Ukrainoj, vosniknet realnaja ugrosa suverenitetu Belarusi«, Belorusskij zhurnal, 5.3.2014, <http://journalby.com/news/esli-rossiya-ustanovit-kontro 1-nad-ukrainoy-pered-belarusyu-vstanet-realnaya-ugroza-poteri-112>. 


\section{Umfragen zur Ukraine-Krise}

Grafik 1: Wie bewerten Sie den Anschluss der Krim an Russland?

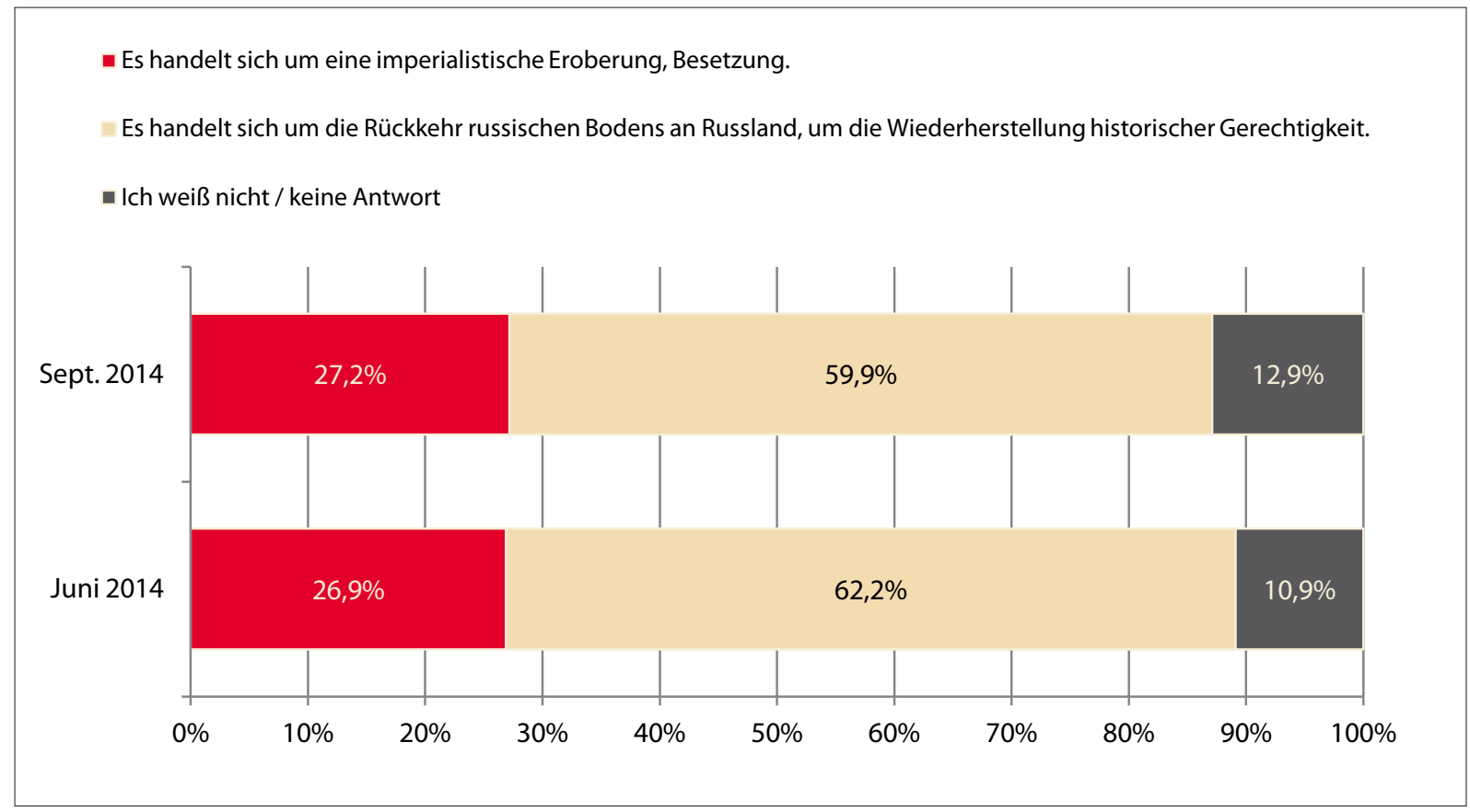

Quelle: IISEPS, Infofokus, September 2014, S. 18, <www.iiseps.org>

Grafik 2: Halten Sie es für möglich, dass Russland analoge Maßnahmen gegenüber Belarus ergreift (d. h., ist eine Annexion eines Teils oder des gesamten Gebiets von Belarus durch Russland möglich)?

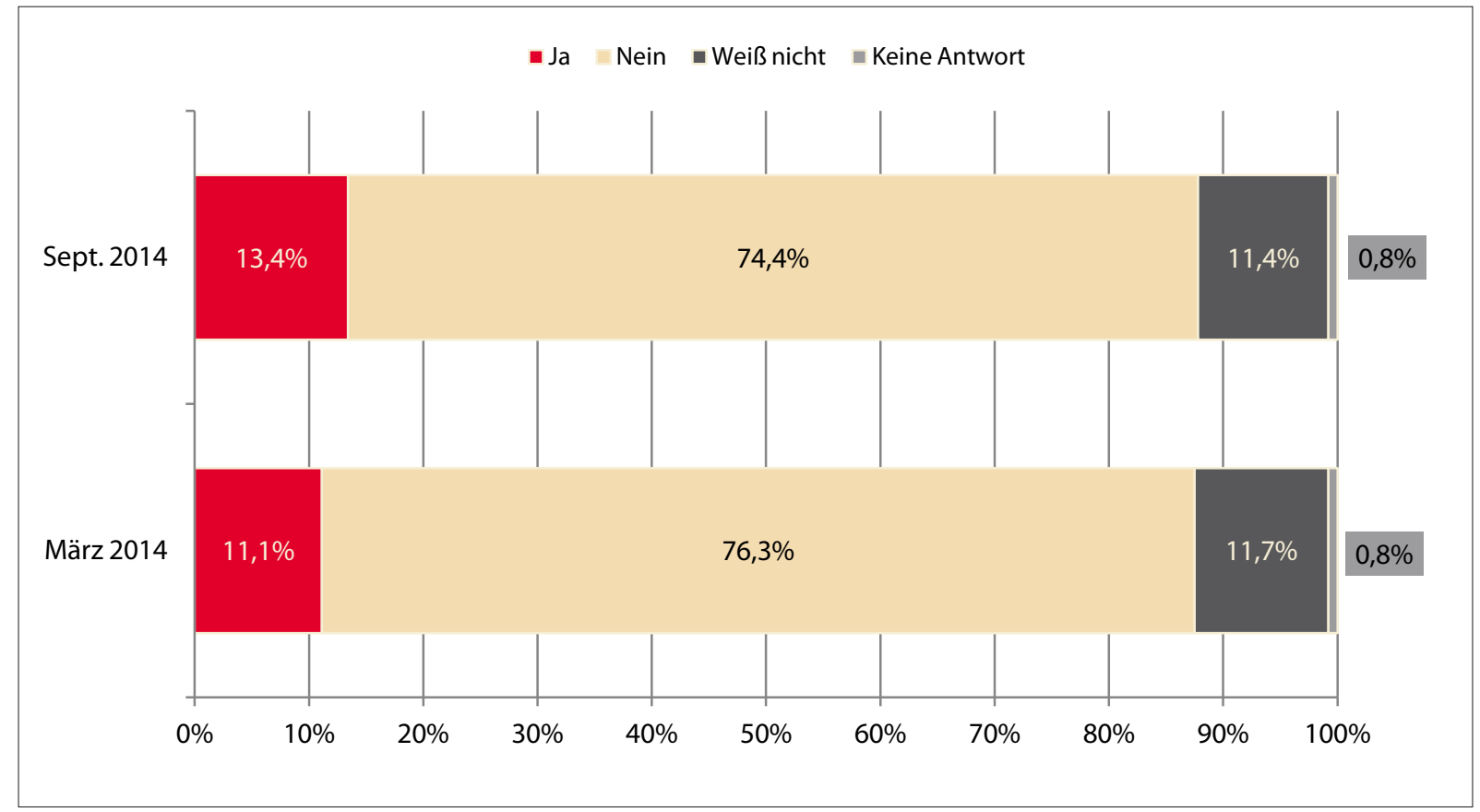

Quelle: Andrej Wardomazkij: Wlijanie ukrainskich sobytij na obschtschestennoe mnenie RB: Rossijskij konzept i belaruskij sasor, in: Belaruskaja Analititscheskaja Masterskaja: Belarus. Realnost, Nr. 14, September 2014, S. 8-15, hier S. 13. 
Grafik 3: Wie bewerten Sie den Einsatz der Streitkräfte durch die ukrainische Führung zur Wiederherstellung der Kontrolle über den Donbass?

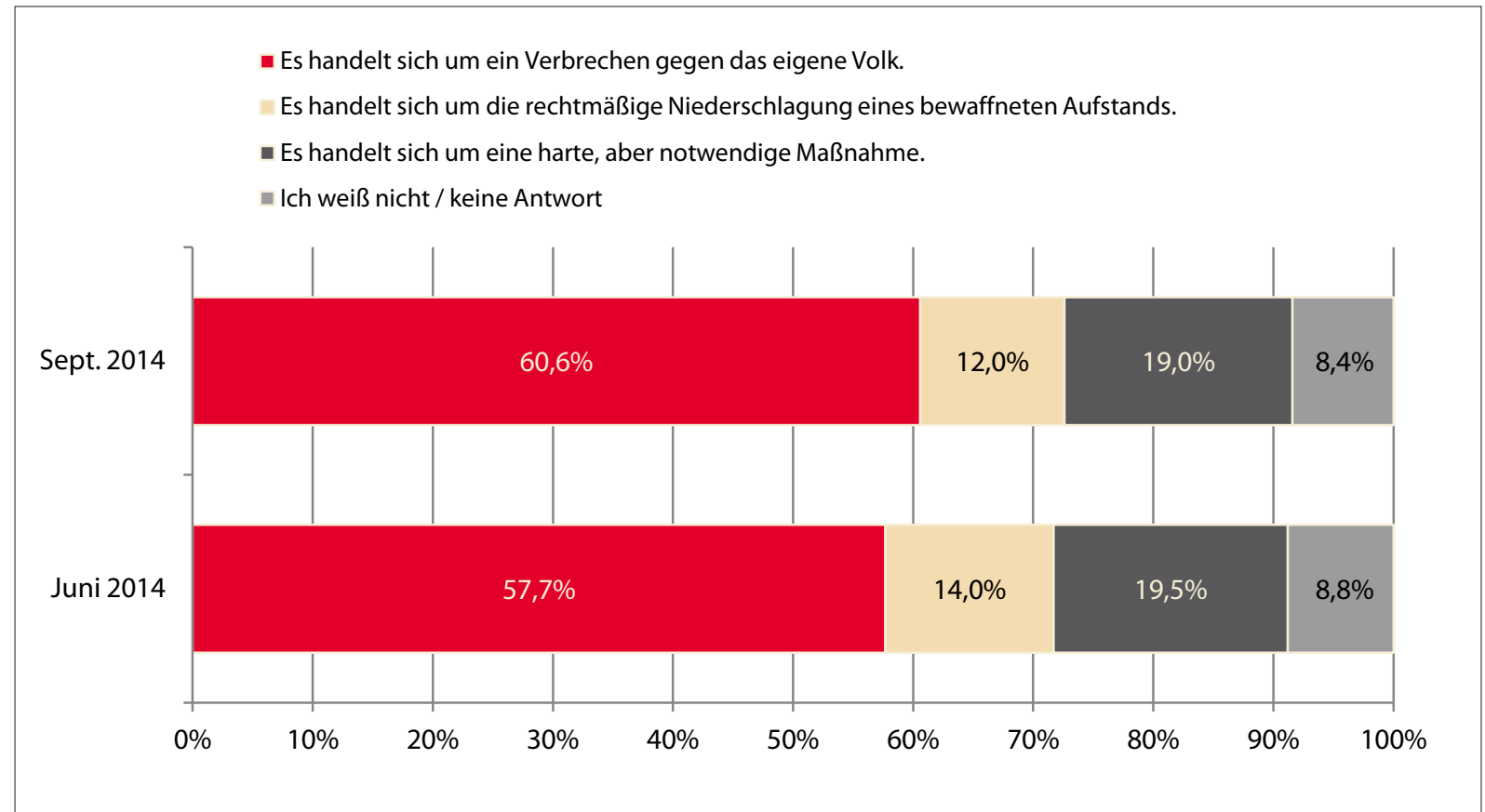

Quelle: IISEPS, Infofokus, September 2014, S. 18, <www.iiseps.org>

Grafik 4: Die westlichen Staaten haben harte Sanktionen gegen Russland wegen der UkrainePolitik verhängt. Wie bewerten Sie diese Maßnahmen?

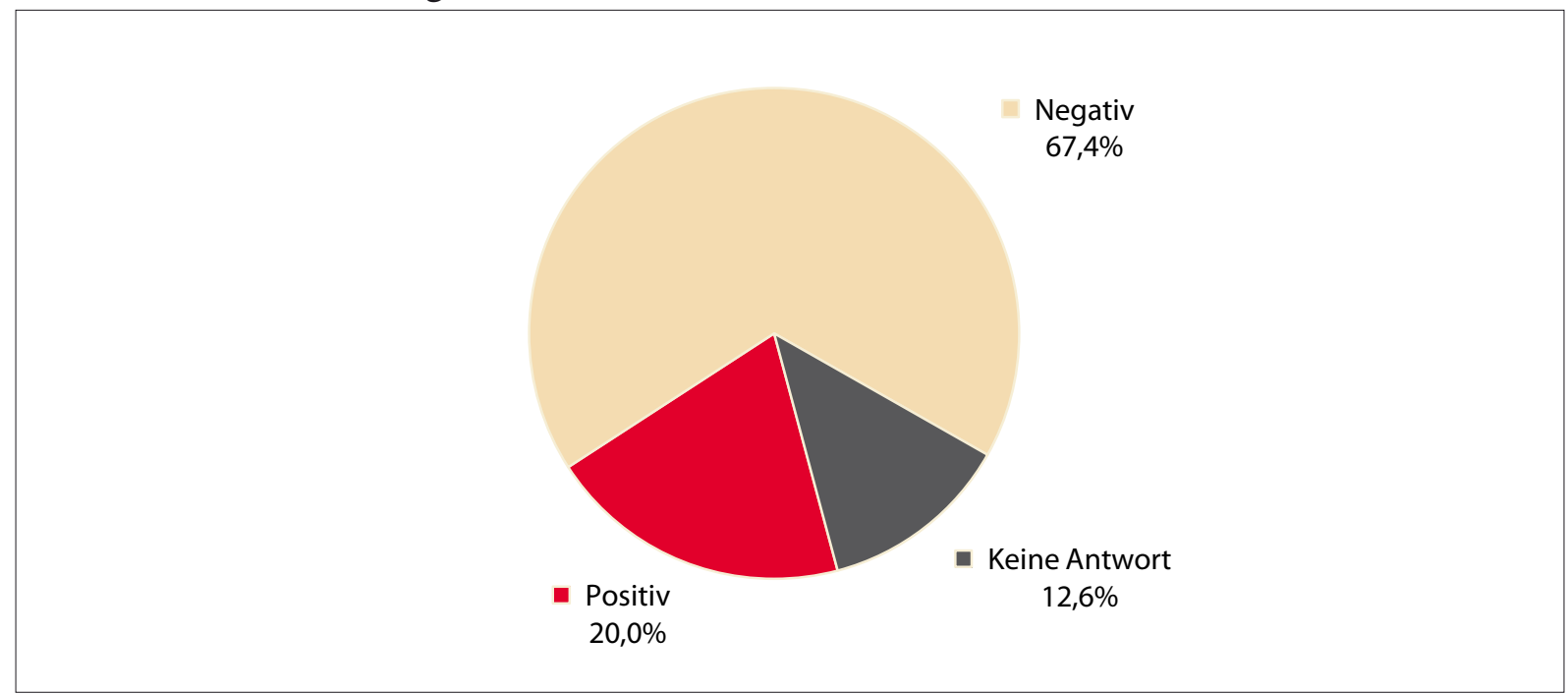

Quelle: IISEPS, Infofokus, September 2014, S. 19, <www.iiseps.org> 
Grafik 5: In Russland vertreten einige die Position, dass Russland seine Armee einsetzen soll, um den Teilnehmern der bewaffneten Proteste in der Ostukraine zu helfen. Wie stehen Sie zu diesem Vorschlag?

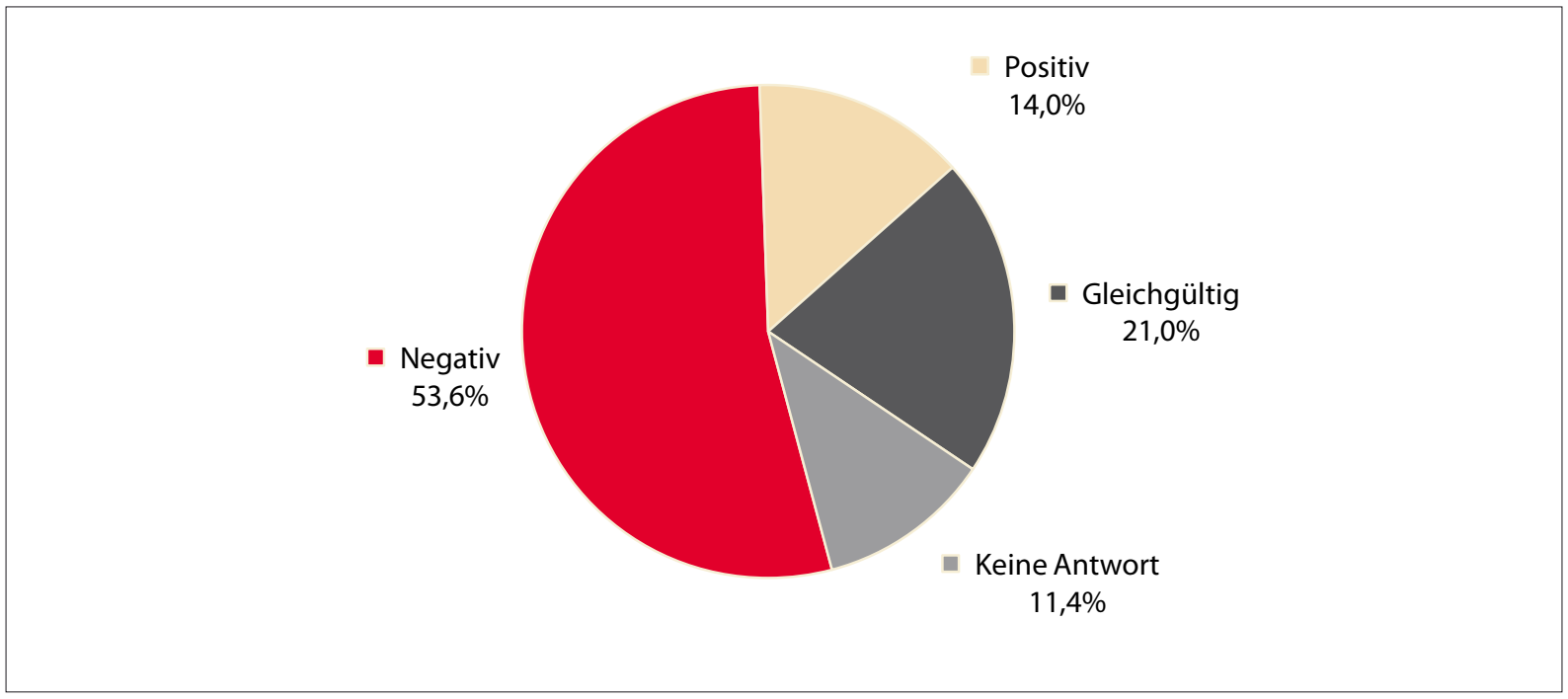

Quelle: IISEPS, Infofokus, September 2014, S. 19, <www.iiseps.org>

Grafik 6: Wenn Russland die Entscheidung treffen würde, seine Truppen in der Ukraine einzusetzen, soll Belarus in diesem Falle Ihres Erachtens nach Russland erlauben, die Truppen über belarussisches Gebiet einmarschieren zu lassen?

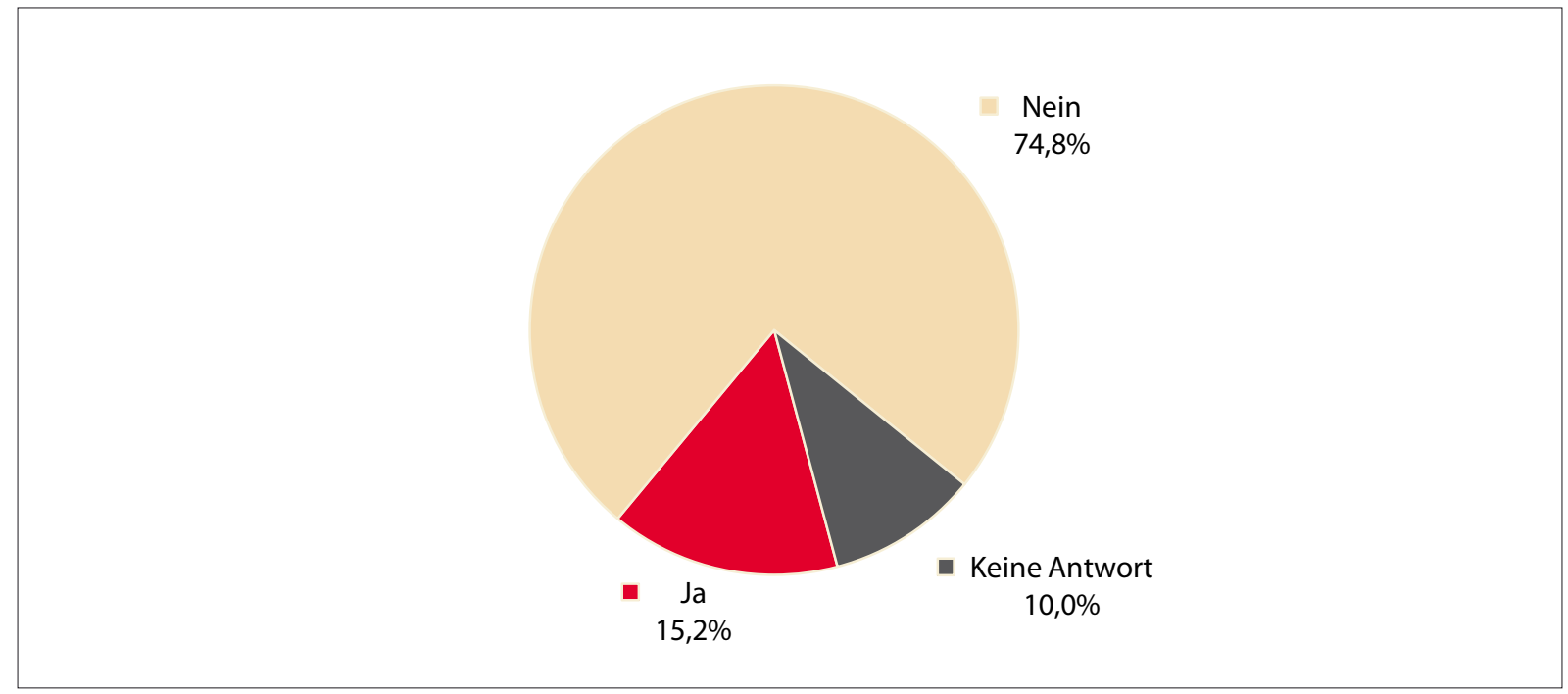

Quelle: IISEPS, Infofokus, September 2014, S. 20, <www.iiseps.org> 
Grafik 7: Wie stehen Sie zur Mitwirkung belarussischer Bürger an den Kampfhandlungen in der Ostukraine?

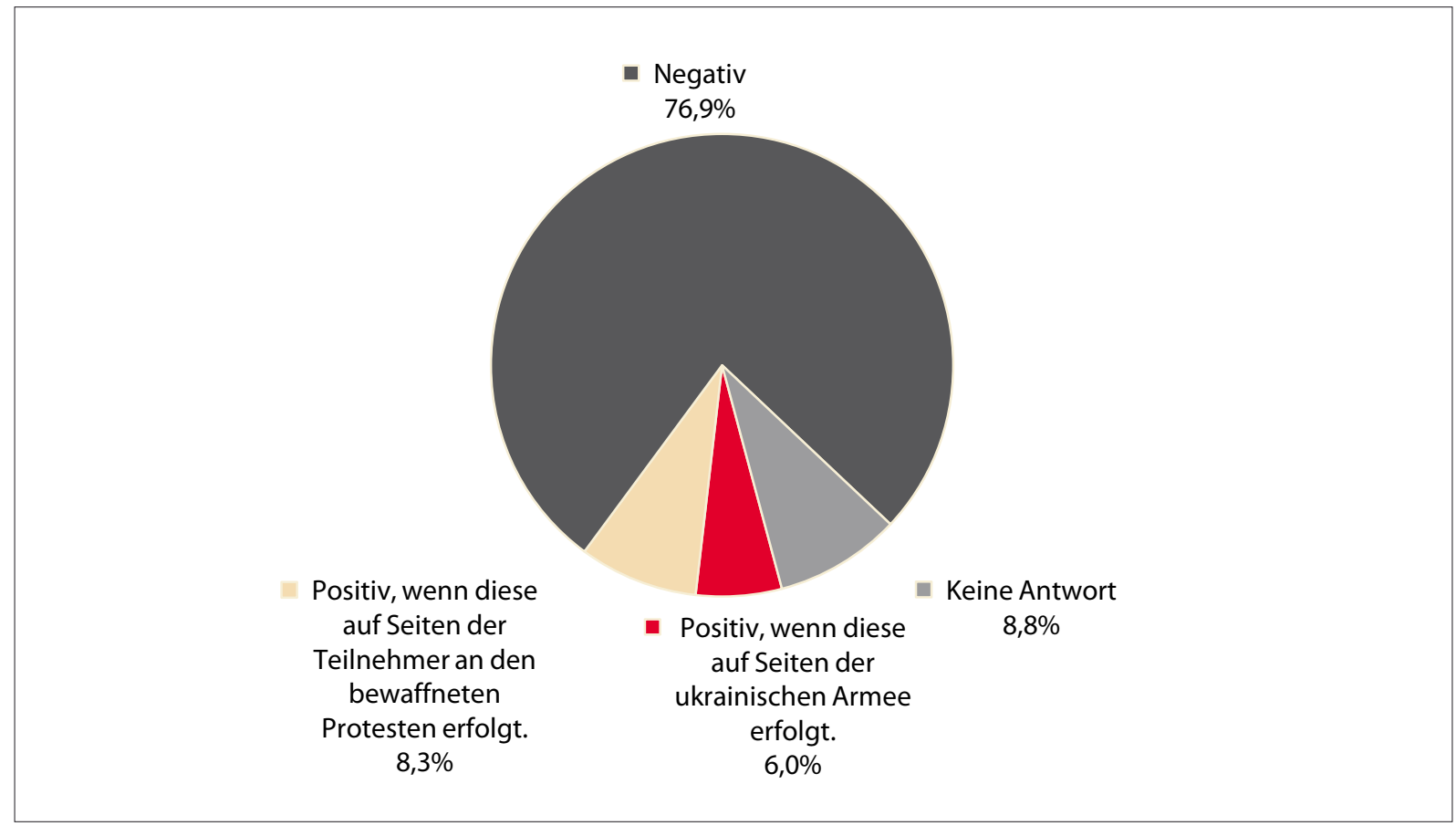

Quelle: IISEPS, Infofokus, September 2014, S. 20, <www.iiseps.org>

Grafik 8: Sind Sie persönlich bereit, Einschränkungen und Schwierigkeiten im Kauf zu nehmen, um Russland zu unterstützen?

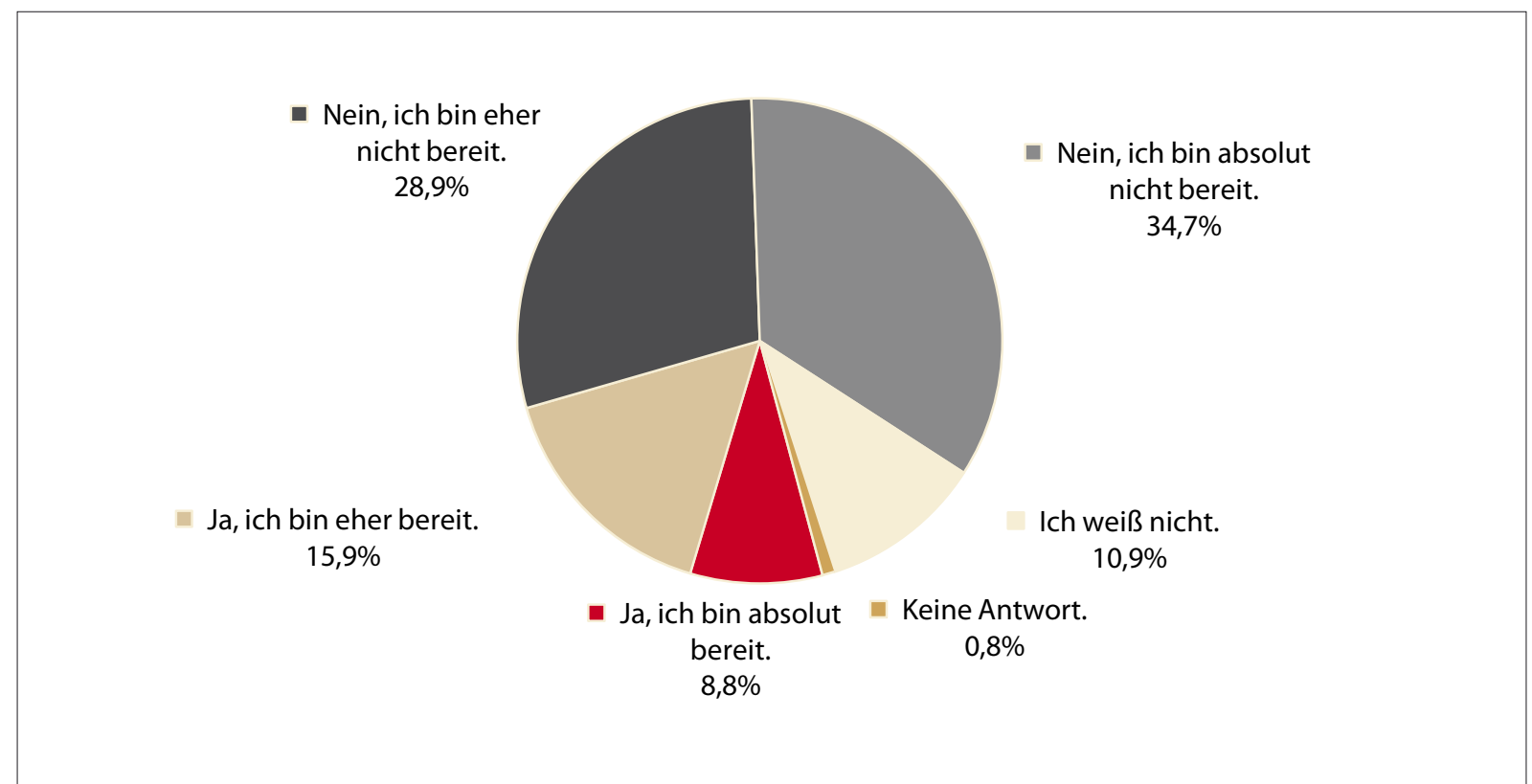

Quelle: Andrej Wardomazkij: Wlijanie ukrainskich sobytij na obschtschestennoe mnenie RB: Rossijskij konzept i belaruskij sasor, in: Belaruskaja Analititscheskaja Masterskaja: Belarus. Realnost, Nr. 14, September 2014, S. 8-15, hier S. 14. 
Grafik 9: Ende Juli fanden in Minsk Verhandlungen zwischen Vertretern der ukrainischen Führung und Vertretern der Teilnehmer an den bewaffneten Protesten in der Ostukraine statt. Wie bewerten Sie den Umstand, dass Belarus die Plattform für Verhandlungen geboten hat?

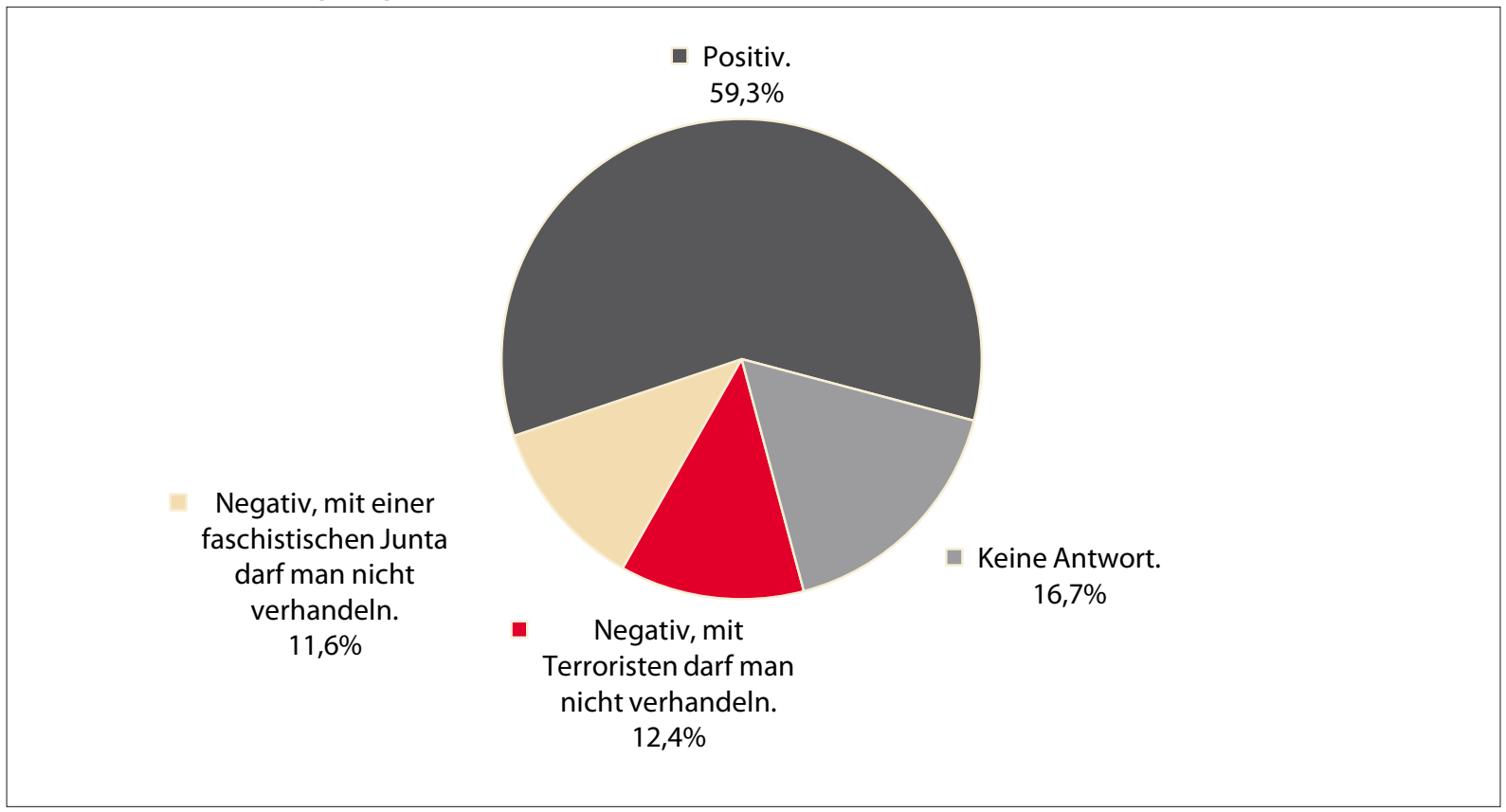

Quelle: IISEPS, Infofokus, September 2014, S. 20, <www.iiseps.org>

Grafik 10: Welche Politik verfolgt Präsident A. Lukaschenka Ihrer Ansicht nach in der UkraineKrise?

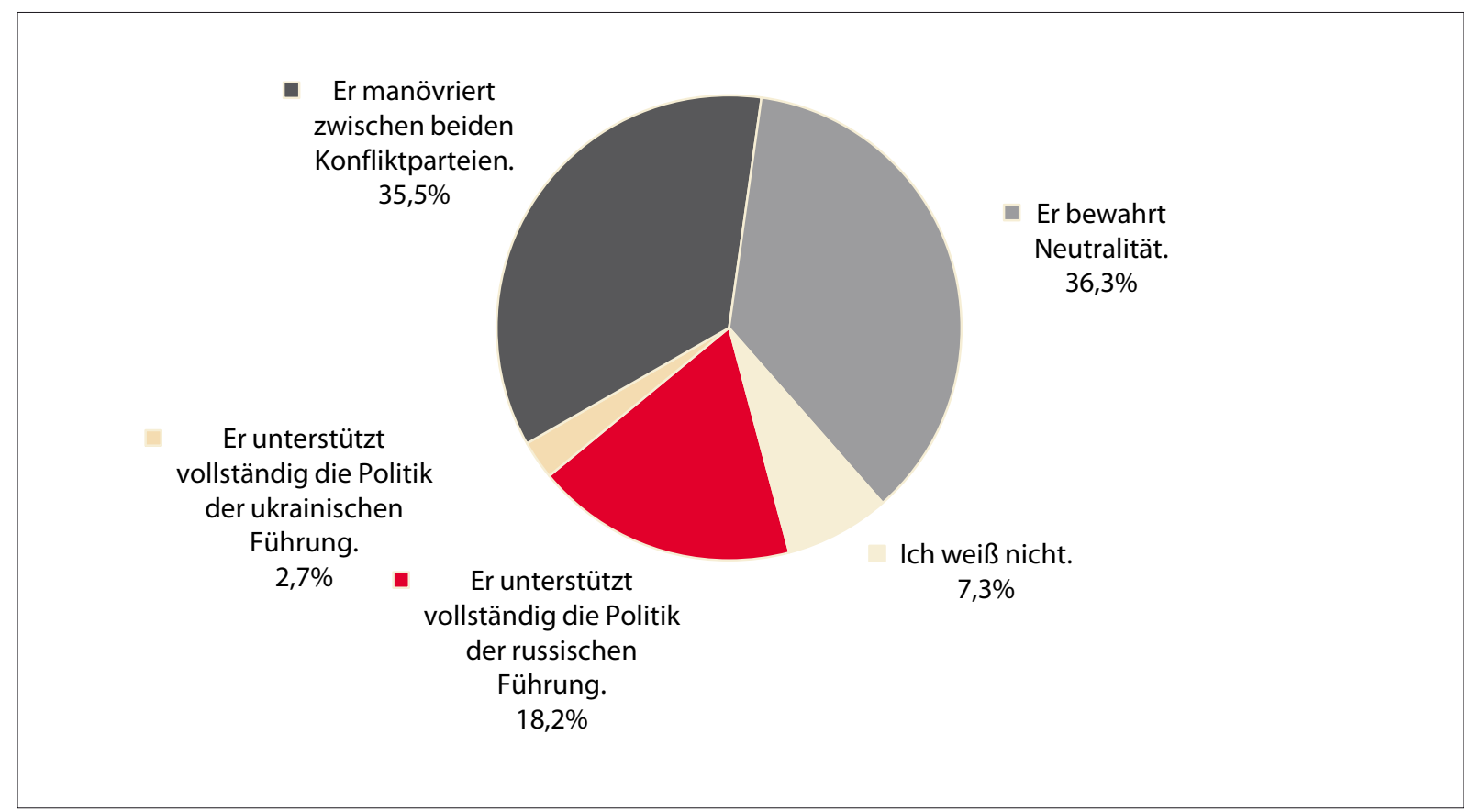

Quelle: IISEPS, Infofokus, September 2014, S. 20, <www.iiseps.org> 
Grafik 11: Wie bewerten Sie die Politik von Präsident Aljaksandr Lukaschenka in der UkraineKrise?

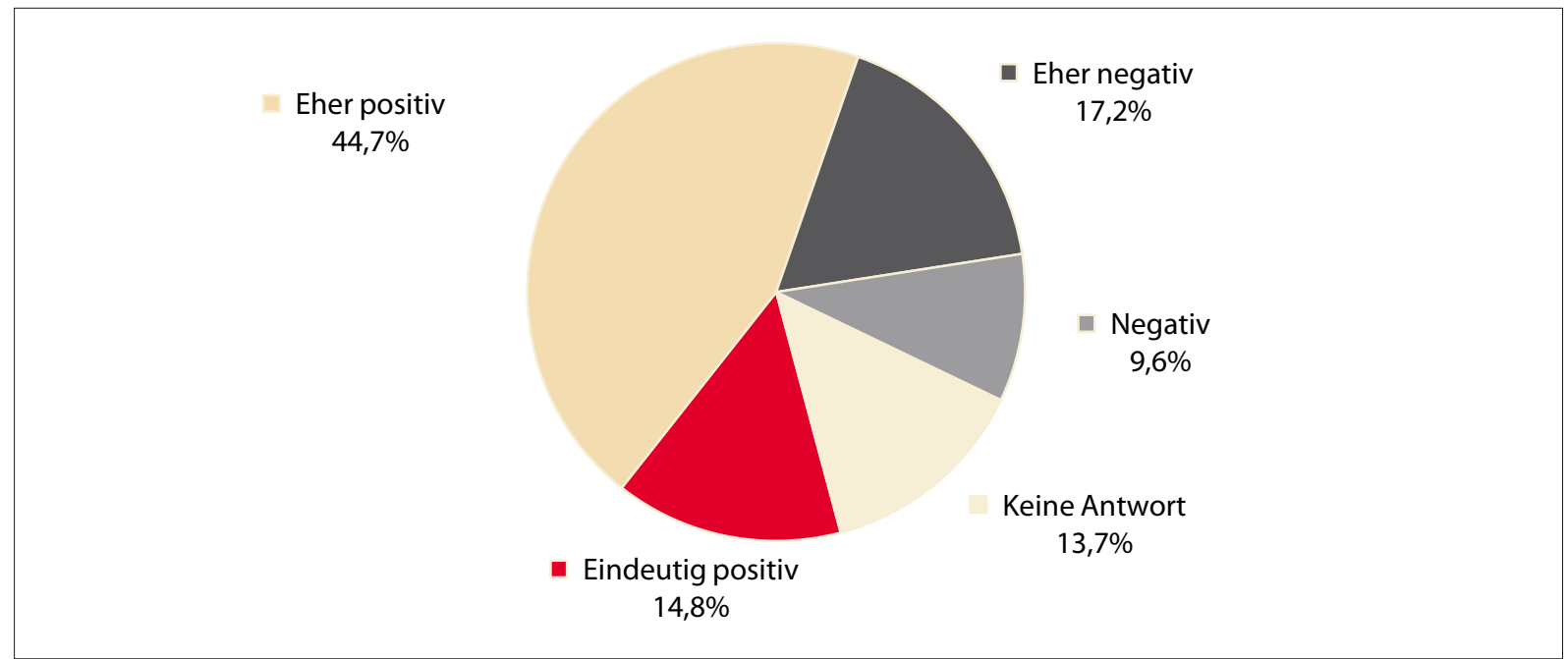

Quelle: IISEPS, Infofokus, September 2014, S. 21, <www.iiseps.org>

\section{Unter Schock: Die belarussische Wirtschaft im Kontext des Ukraine-Konflikts}

Robert Kirchner, Berlin

\section{Zusammenfassung:}

Der Ukraine-Konflikt hat erhebliche indirekte Auswirkungen auf die belarussische Wirtschaft. Insbesondere leiden die Exporte unter der Stagnation des wichtigsten Handelspartners (Russland) sowie unter der starken Rezession beim zweitwichtigsten Partner (Ukraine). Aber auch die westlichen Sanktionen gegen Russland belasten die Finanzströme und damit das Finanzierungsumfeld in Belarus. Auch wenn sich bestimmte Bereiche, z. B. der Lebensmittelexport nach Russland positiv entwickeln dürften, so ist insgesamt mit einer deutlichen negativen Auswirkung des Ukraine-Konflikts auf Belarus auszugehen.

Diese Entwicklung zeigt deutlich die Risiken die sich ergeben, wenn ein Land (Belarus) wirtschaftlich zu stark von einem einzigen Land (Russland) abhängt. Belarus sollte hieraus Lehren ziehen und eine Diversifizierung seiner Außenwirtschaft vorantreiben. Ein IWF-Programm würde die finanziellen Ströme und ein WTO-Beitritt den Handel diversifizieren und stabilisieren. Aber auch eine engere Zusammenarbeit mit internationalen Finanzinstitutionen wäre sinnvoll, um beispielweise mit Hilfe von Pre-Privatisierungen Strukturreformen zu implementieren.

\section{Aktuelles Umfeld geprägt von externen Schocks}

Belarus befindet sich gegenwärtig in einem in wirtschaftlicher und geopolitischer Hinsicht sehr angespannten regionalen Umfeld. Dabei kann man vier verschiedene Bereiche unterscheiden, in denen aktuell
Schocks auftreten und welche die wirtschaftliche Situation von Belarus signifikant beeinflussen.

1. Wirtschaftliche Lage in Russland: Es lässt sich gegenwärtig ein deutlicher Abschwung der russischen Volkswirtschaft beobachten, die in diesem Jahr im Prinzip stagnieren wird (IWF-Prognose: 0,2 \% reales 
BIP-Wachstum). Auch 2015 wird mit 0,5\% Wachstum keine wesentliche Trendwende erreicht. Der aktuell zu beobachtende Ölpreisverfall kommt als zusätzlicher belastender Faktor hinzu.

2. Wirtschaftliche Lage in der Ukraine: Das BIP wird in diesem Jahr um prognostizierte $6,5 \%$ zurückgehen. Im besten Fall gibt es laut IWF 2015 einen geringen Anstieg um $1 \%$; ein weiterer Rückgang ist aber durchaus denkbar.

3. Sanktionen des Westens gegen Russland: Diese betreffen verschiedene Bereiche, wobei die Maßnahmen im Finanzsektor sicher die stärksten Wirkungen entfachen.

4. Gegensanktionen Russlands: Als Reaktion auf die westlichen Sanktionen hat Russland seinerseits Handelsbeschränkungen erlassen, insbesondere beim Import von Lebensmitteln.

Im Folgenden werden die zu erwartenden Auswirkungen dieser Schocks auf das Land beschrieben. Dafür analysieren wir zuerst die außenwirtschaftlichen Verflechtungen des Landes, bevor wir uns dann den entsprechenden Auswirkungen zuwenden.

\section{Außenwirtschaftliche Verflechtungen von Belarus}

Grundsätzlich lassen sich die internationalen Verflechtungen in die Bereiche Außenhandel und Kapitalverkehr unterteilen. Belarus ist im Außenhandel eine sehr offene Volkswirtschaft; der Offenheitsgrad (Verhältnis Außenhandel zu BIP) liegt bei sehr hohen $112 \%$.

Auffällig ist der hohe Anteil von Russland am Warenhandel, der bei über $50 \%$ liegt. Auch die Ukraine spielt mit $8 \%$ eine hervorgehobene Rolle als Handelspartner. Auf Länderbasis (d. h. wenn die Mitgliedsländer der EU einzeln betrachtet werden) ist die Ukraine nach Russland der zweitwichtigste Handelspartner von Belarus. Neben dem Warenhandel spielt für Belarus auch der Dienstleistungshandel eine wichtige Rolle. Das Land ist ein wichtiges Transitland für russisches Öl und Gas in die EU, bzw. für den Güterverkehr zwischen beiden Handelspartnern.

Bei der Betrachtung des Kapitalverkehrs setzt sich die prominente Rolle Russlands fort. So stammen 59\% des Bestands an ausländischen Direktinvestitionen aus Russland, häufig sind die Kapitalgeber staatliche russische Unternehmen. Auch im Bankensektor herrscht ein starker russischer Einfluss. Zwar sind staatliche belarussische Banken dominant (ca. $65 \%$ Markanteil), aber russische staatliche Banken haben einen signifikanten Anteil von $25 \%$. Den restlichen Markt teilen sich private Banken.

Es muss darüber hinaus betont werden, dass Belarus seitens Russlands signifikante finanzielle Unterstüt- zung erhält, die über verschiedene Kanäle gewährt werden (z. B. verbilligter Bezug von Öl und Gas, Kredite zu Vorzugskonditionen). Diese belaufen sich Schätzungen zufolge auf ca. 7,7 Mrd. US-Dollar pro Jahr, d. h. auf mehr als $10 \%$ des BIP. Im Gegenzug ist Belarus Mitglied in der Zollunion. Zusammenfassend ist festzuhalten, dass Belarus in außenwirtschaftlicher Hinsicht wenig diversifiziert ist und in hohem Maße von Russland abhängt.

\section{Implikationen des Ukraine-Konflikts für Belarus}

Im Handelsbereich stehen eine Ausweitung der Lebensmittelproduktion und deren vermehrter Export nach Russland im Fokus von Politik und Öffentlichkeit. Tatsächlich sind nach vorliegenden Zahlen diese bereits angestiegen. Die Frage ist aber, ob dies ein längerfristiger Trend ist, der auch seitens Russlands akzeptiert wird. Auch müssen die Auswirkungen solcher Exportsteigerungen auf die Inflation in Belarus (aktuell fast $20 \%$ ) bedacht werden. Im Bereich Maschinenbau, wo Russland der wichtigste Absatzmarkt ist, sieht die Entwicklung dagegen sehr problematisch aus. Einerseits dämpft der trübe Wirtschaftsausblick die Investitionsneigung, andererseits sorgen die bereits wirkenden Finanzsanktionen für höhere Zinsen; beides dämpft den Import von entsprechenden Ausrüstungen und Maschinen in Russland. Zusätzlich belastet die Krise in der Ukraine den belarussischen Warenexport - die Importe der Ukraine sind im Jahresverlauf um über $25 \%$ gegenüber dem Vorjahr zurückgegangen. Ein zusätzliches Problem für die Wettbewerbsfähigkeit von Belarus deutet sich dabei an: So hat der belarussische Rubel seit Jahresmitte gegenüber dem russische Rubel um $34 \%$ und gegenüber der Hryvnia um $22 \%$ aufgewertet.

Beim Kapitalverkehr ist zu vermuten, dass Finanzsanktionen gegen bestimmte russische Banken deren Refinanzierung einschränken bzw. verteuern, und damit auch deren belarussische Töchter betroffen sind.

Insgesamt sind diese Entwicklungen negativ zu bewerten. Darüber hinaus stellt sich die zentrale Frage, ob im beschriebenen Umfeld die russ. Subventionen weiterhin so fließen werden wie bisher, oder ob es diesbezügliche Einschränkungen der Bereitschaft und/oder Fähigkeit seitens Russlands gibt.

\section{Strategien zur Begrenzung der Auswirkungen}

Die belarussischen Entscheidungsträger sollten überlegen, wie man die zu erwartenden negativen wirtschaftlichen Auswirkungen reduzieren kann. Da insbesondere die hohe Abhängigkeit von Russland dafür ursächlich ist, sollten entsprechende Überlegungen hier ansetzen. 
Eine Strategie sollte dabei aus mehreren Komponenten bestehen.

1. Makroökonomische Stabilisierung: Die Situation ist unverändert schwierig, wie die hohen externen Defizite, der geringe Bestand an Devisenreserven, sowie der Druck auf den Wechselkurs dokumentieren. Ein IWF-Stabilisierungsprogramm als externer Anker wäre in diesem Umfeld anzuraten, um einerseits eine günstige Finanzierung der externen Defizite sicherzustellen, vor allem aber um einen Abbau dieser Defizite durch Politikkorrekturen zu erreichen.

2. Außenhandel: Eine zentrale Botschaft liegt in der Diversifizierung des Außenhandels in Bezug auf die Handelspartner. Hier kann die angestrebte WTOMitgliedschaft neue Chancen eröffnen. Belarus sollte auch eruieren, ob sich durch die Freihandelsabkommen (DCFTA) der Ukraine, Moldau und Georgien mit der EU eventuell auch neue Chancen, etwa durch Joint-Ventures, ergeben.

3. Strukturreformen: Die Diversifizierung im Finanzbzw. Bankensektor ist sicherlich nicht einfach zu vollziehen. Eine Möglichkeit wäre aber, mit internationalen Finanzinstitutionen über eine Pre-Privatisierung von staatlichen Banken zu reden. Dies würde den Sektor diversifizieren, frisches Kapital zur Entwicklung der Banken generieren, und damit der Stabilität des Finanzsektors dienen.

\section{Fazit}

Die starke wirtschaftliche Anbindung an Russland impliziert im aktuell schwierigen wirtschaftlichen Umfeld gewisse Risiken für Belarus; ein Umdenken ist daher erforderlich. Eine Kombination aus einer makroökonomischen Stabilisierung sowie handelspolitischen Reformen und Strukturreformen erscheint sinnvoll, um negative Auswirkungen auf das Land zu begrenzen.

Entsprechende glaubwürdige Reformbemühungen sollten durch Deutschland bzw. die EU unterstützt werden, helfen sie doch, das Land zu diversifizieren, und damit widerstandsfähiger gegenüber negativen wirtschaftlichen Schocks zu machen.

\section{Über den Autor:}

Robert Kirchner ist Koordinator des German Economic Team Belarus (GET Belarus); dieses führt seit 2003 einen wirtschaftspolitischen Dialog mit reformorientierten Entscheidungsträgern der belarussischen Regierung. Es wird im Rahmen des TRANSFORM-Nachfolgeprogramms der Bundesregierung durch das Bundesministerium für Wirtschaft und Energie finanziert.

Der abgedruckte Text ist ein Nachdruck des aktuellen Newsletters des German Economic Team Belarus (Nr. 31 vom November 2014). Zum regelmäßigen Bezug des Newsletters genügt eine E-Mail an <newsletter@get-belarus.de>.

\section{Lesetipps:}

- Belarus' state apparatus shows high level of coordination in food war with Russia, Belarus in focus, 9.12.2014, $<$ belarusinfocus.info/p/6515>.

- World Bank: Belarus Economic Update, November 2014, <www.worldbank.org/content/dam/Worldbank/docu ment/eca/belarus/BY_Macro_Update_November_2014.pdf $>$.

- EBRD Office of the Chief Economist: In the Heavy Shadow of the Ukraine/Russia Crisis. Regional Economic Prospects in EBRD Countries of Operation: September 2014, <http://www.ebrd.com/downloads/research/REP/ rep-2014.pdf>. 


\section{BIP, Wechselkurs, Im- und Export}

Grafik 1: Reales BIP-Wachstum von Belarus, der Ukraine und Russland

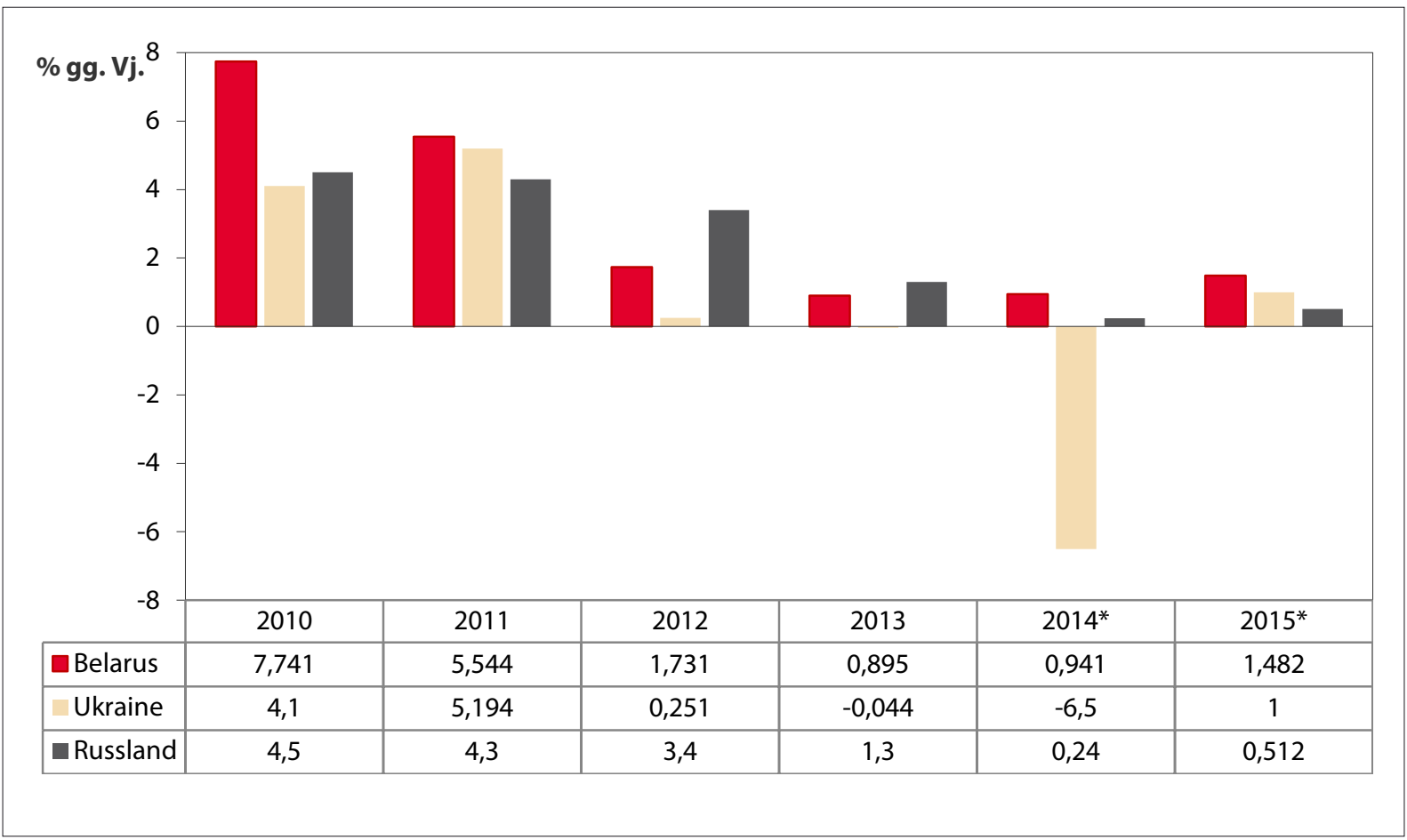

* Schätzung bzw. Prognose

Quelle: Internationaler Währungsfonds, World Economic Outlook Oktober 2014, S. 60, <http://www.imf.org/external/pubs/ft/ weo/2014/02/pdf/text.pdf>

Grafik 2: Wechselkurs BYR, UAH und RUB gegenüber USD, indexiert, 1. Jan. $2014=100$

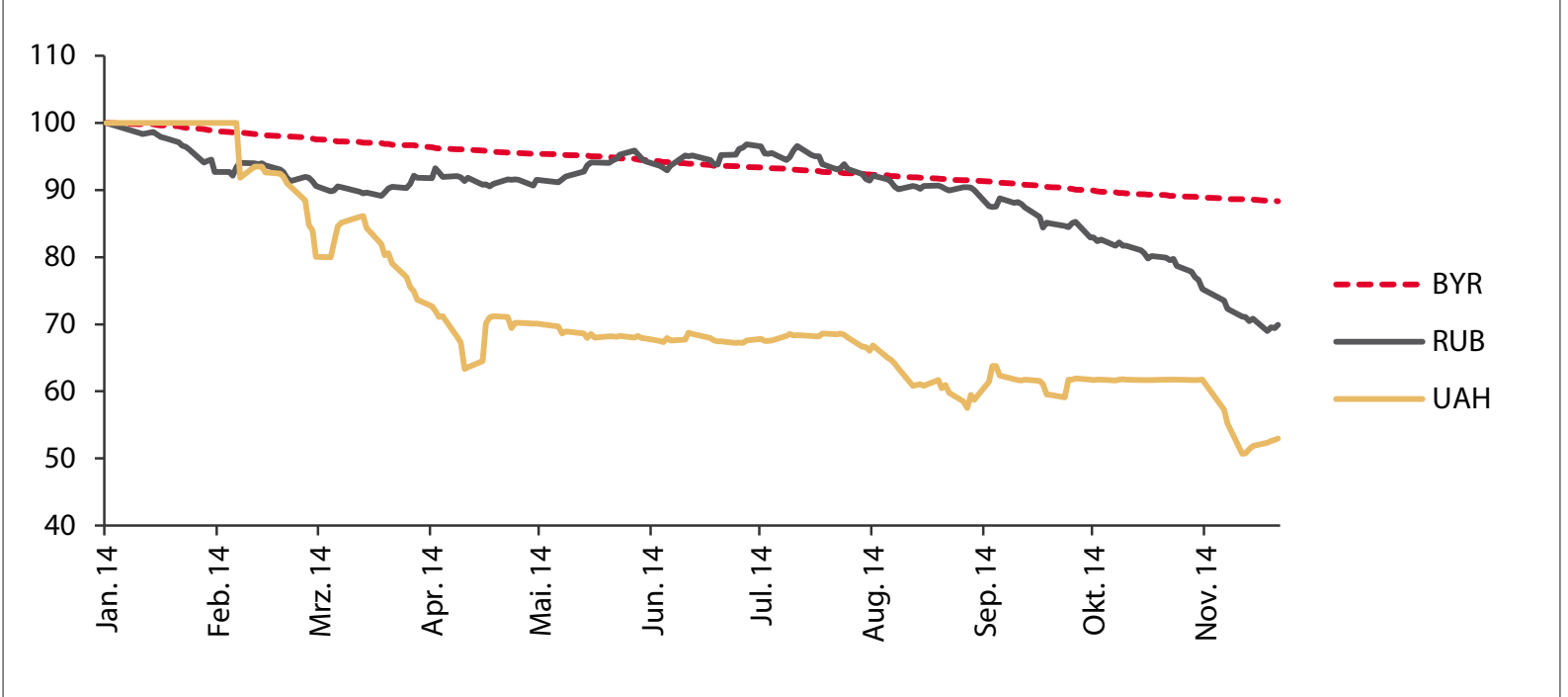

Quelle: Nationalbanken von Belarus (<http://www.nbrb.by>), Russland (<http://www.cbr.ru〉) und Ukraine (<http://www.bank. gov.ua>) 
Grafik 3: Struktur der ausländischen Direktinvestitionen vom 1. Januar 2014

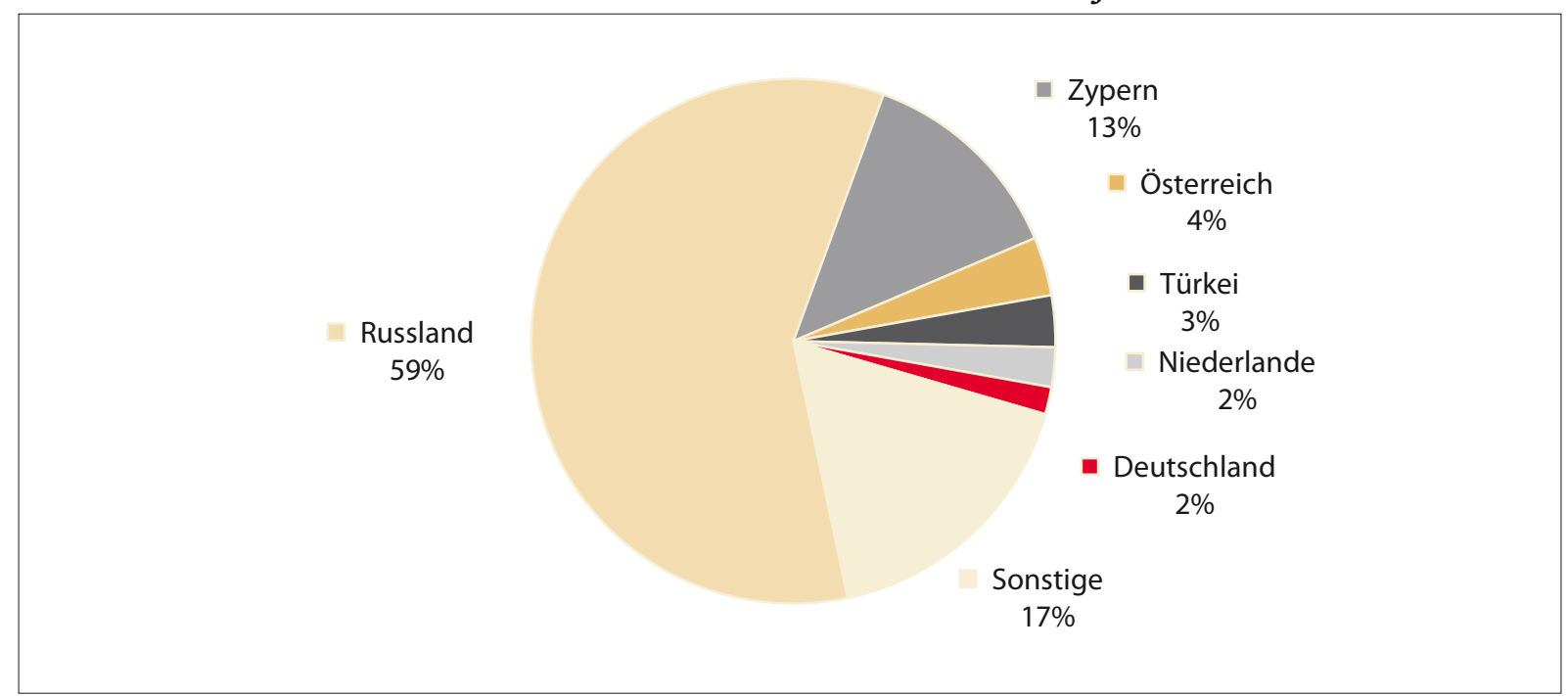

Quelle: Nationalbank der Republik Belarus, <http://www.nbrb.by/engl/statistics/ForeignDirectInvestments/>

Grafik 4: Warenhandel nach Regionen 2013

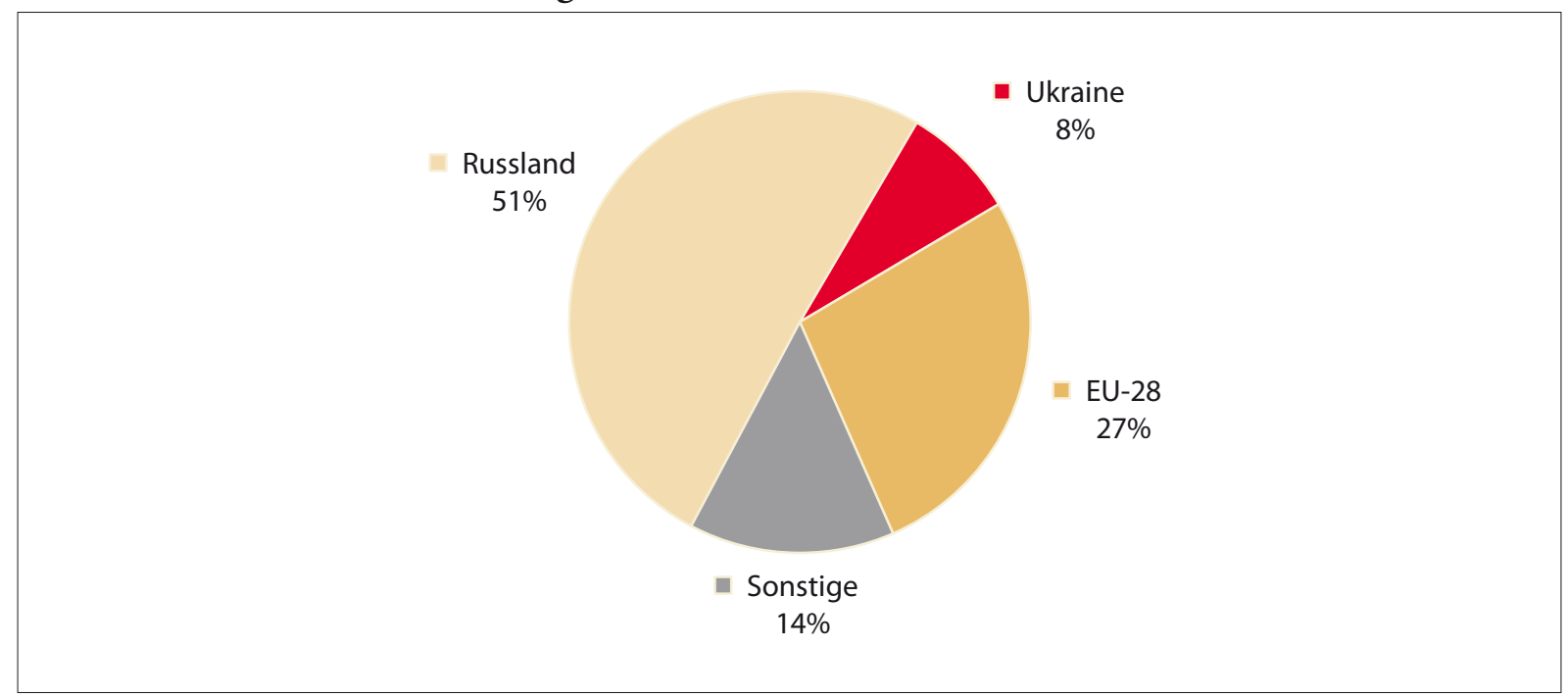

Quelle: UN Comtrade, <http://comtrade.un.org/> 
Grafik 5: Warenexporte nach Ländern 2013

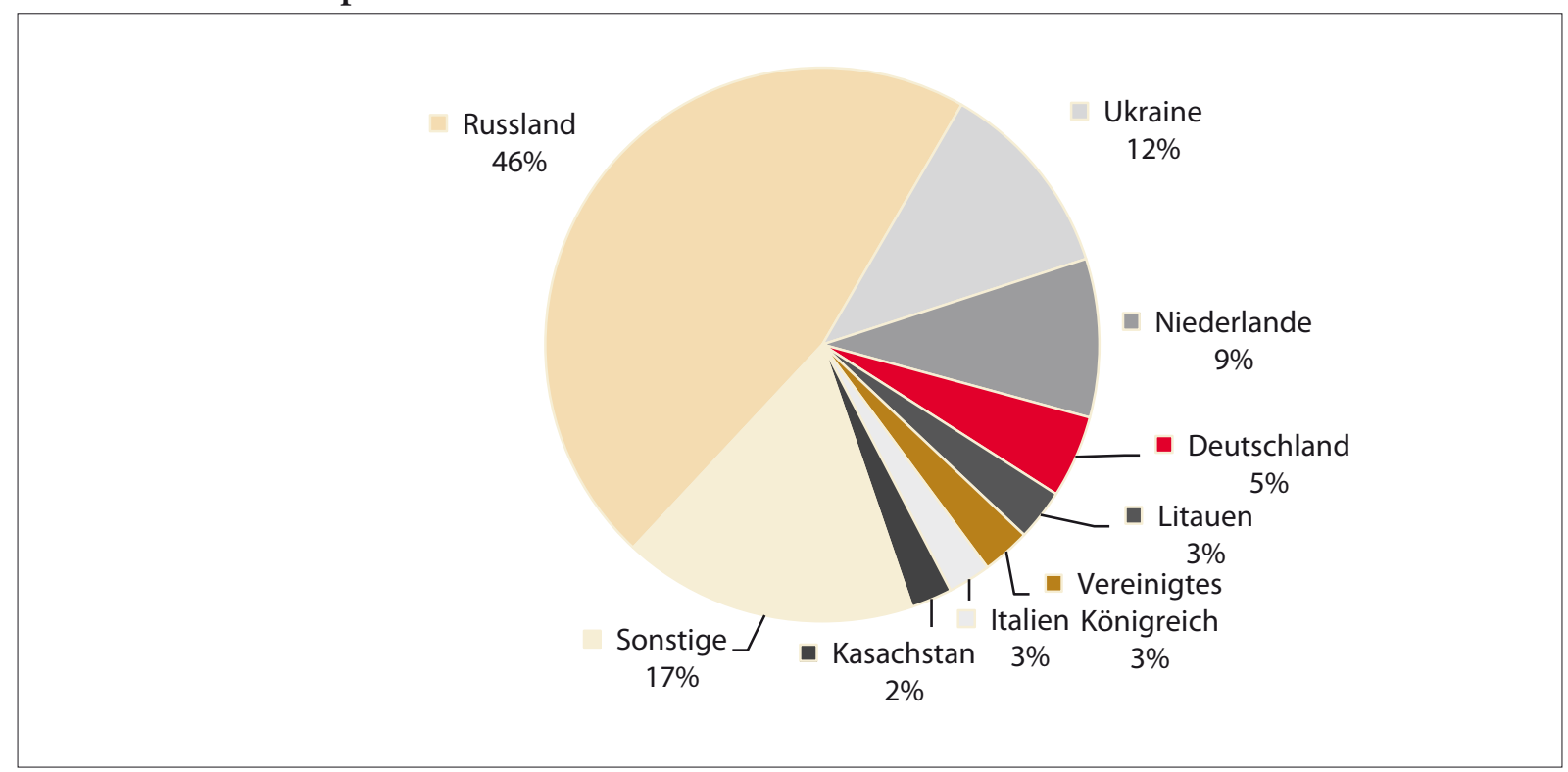

Quelle: UN Comtrade, <http://comtrade.un.org/>

Grafik 6: Warenimporte nach Ländern 2013

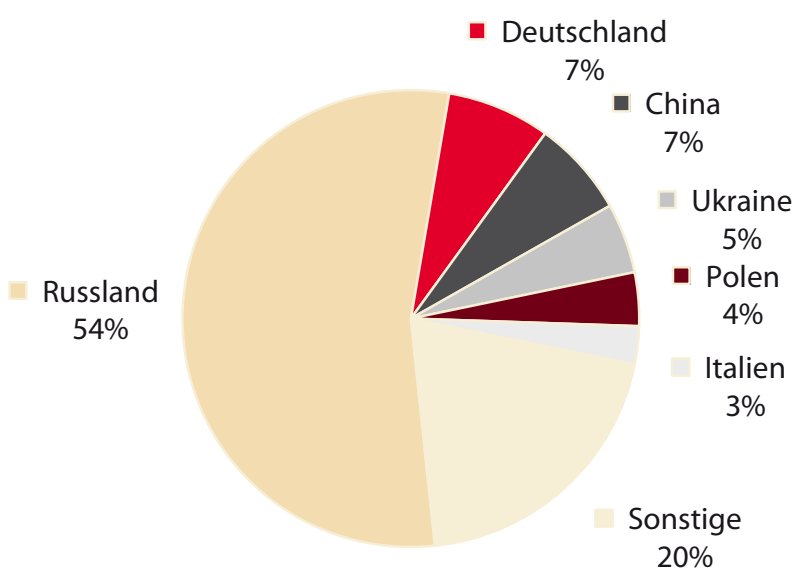

Quelle: UN Comtrade, <http://comtrade.un.org/> 


\section{Zur Lage ukrainischer Flüchtlinge in Belarus}

Dem UN-Nothilfekoordinator (OCHA) zufolge hielten sich 60.261 Menschen aus der Ukraine zum 12. Dezember 2014 in Belarus auf (siehe Karte unter <http://reliefweb.int/sites/reliefweb.int/files/resources/Ukraine\%20Displace ment\%20Map\%20-\%2012\%20December.pdf >). In Russland hatten zur selben Zeit 465.721 Ukrainer Zuflucht gefunden, in allen Nachbarstaaten zusammen befanden sich insgesamt 567.956 ukrainische Flüchtlinge. Gleichzeitig gab es in der Ukraine selber 542.080 Binnenflüchtlinge. Damit haben sich 5,4\% der Menschen, die wegen des Kriegs aus ihrer Heimat in den Gebieten Donezk und Luhansk geflohen sind, auf dem Weg nach Belarus gemacht. Die folgende Dokumentation gibt einen Einblick, wie die ukrainischen Flüchtlinge in Belarus aufgenommen werden. Dabei ist auffällig, welchen großen Stellenwert neben der humanitären Aspekte demographische und Beschäftigungsfragen haben.

Die Redaktion

\section{Informationen des belarussischen Innenministeriums für Bürger der Ukraine, die nach Belarus kommen}

[...]

In den ersten acht Monaten des laufenden Jahres haben 531 Ausländer, darunter 403 ukrainische Staatsbürger, den Flüchtlingsstatus oder zusätzlichen Schutz in der Republik Belarus beantragt. Bis zum 1.9.2014 hat das Departement für Staatsbürgerschaft und Migration zu 17 Anträgen ukrainischer Bürger eine Entscheidung getroffen. Drei ukrainische Staatsbürger, die aus den Gebieten Donezk und Luhansk der Ukraine gekommen sind, haben zusätzlichen Schutz erhalten. Ein Fall wurde auf Antrag des Ausländers eingestellt und 13 Bürgern der Ukraine wurde kein zusätzlicher Schutz gewährt.

\section{Spezifika der Arbeitssuche}

Laut Präsidialerlass Nr. 420 vom 30. August 2014 „Über den Aufenthalt von Staatsbürgern der Ukraine in der Republik Belarus« müssen belarussische Arbeitgeber, die ukrainische Bürger (aus den Gebieten Donezk und Luhansk) beschäftigen, keine Abgabe an den Staat zahlen, um die Genehmigung für die Beschäftigung von ausländischen Arbeitskräften sowie die Arbeitserlaubnis für die Mitarbeiter zu erhalten. Die Bearbeitungsfrist für die Ausstellung der Arbeitserlaubnis wurde zudem von 15 auf 5 Tage verkürzt.

[...]

Um die Suche nach einer geeigneten Arbeit für unterschiedliche Bürgergruppen operativ zu unterstützen, hat das Ministerium für Arbeit und Sozialschutz eine Landesweite Datenbank der freien Stellen (im Weiteren: Datenbank) geschaffen. In dieser Datenbank finden sich Informationen über freie Arbeitsplätze sowie Informationen über Organisationen, die freie Stellen haben und ihren Mitarbeitern Wohnraum zur Verfügung stellen. Die Informationen in der Datenbank werden täglich aktualisiert. Die Datenbank ist zugänglich unter: < http://vacancy.mintrud.by/user/ Pages/Public/Main.aspx>.

[...]

Die Anzahl der Sondergenehmigungen für belarussische Arbeitgeber zur Beschäftigung von Staatsbürgern der Ukraine wächst täglich. Während in den ersten sechs Monaten des Jahres 2013844 Sondergenehmigungen erteilt wurden, beläuft sich ihre Zahl 2014 bis zum 8. September auf 10.685 (davon 3.246 in der Stadt Minsk, 2.222 im Gebiet Brest, 1.833 im Gebiet Gomel, 1.652 im Gebiet Minsk, 844 im Gebiet Grodno, 582 im Gebiet Mogiljow und 306 im Gebiet Witebsk).

\section{Spezifika des Erhalts der befristeten und der ständigen Aufenthaltserlaubnis}

Gegenwärtig lässt sich ein anhaltender Trend der Zunahme ukrainischer Bürger, die aus den Gebieten Donezk und Luhansk in die Republik Belarus kommen, beobachten. Ein Teil dieser Bürger hat einen berechtigten Anspruch auf eine ständige oder befristete Aufenthaltserlaubnis (seit April 2014 haben 3.719 ukrainische Staatsbürger eine befristete Aufenthaltserlaubnis erhalten und 2.326 ukrainische Staatsbürger eine ständige Aufenthaltserlaubnis). 
Die Bestimmungen des Präsidialerlasses Nr. 420 vom 30. August 2014 »Über den Aufenthalt von Staatsbürgern der Ukraine in der Republik Belarus" gewähren den ukrainischen Bürgern und Staatenlosen, die zuvor mindestens ein Jahr in den Gebieten Donezk und Luhansk der Ukraine gelebt haben, einige Begünstigungen beim Erhalt einer befristeten oder ständigen Aufenthaltserlaubnis. So werden die zur genannten Gruppe gehörenden Personen befreit von:

- der Bezahlung der Gebühren für den Erhalt der befristeten Aufenthaltserlaubnis in der Republik Belarus, den Erhalt der ständigen Aufenthaltserlaubnis in der Republik Belarus und die Zuteilung des Einwohnertitels;

- der Vorlage von Dokumenten, die für die Erteilung der befristeten oder ständigen Aufenthaltserlaubnis oder des Einwohnertitels von ukrainischen Bürgern eingereicht werden müssen, wenn die Vorlage dieser Dokumente aus objektiven Gründen nicht möglich ist;

- der Bezahlung der medizinischen Dienstleistungen, welche die Einrichtungen des staatlichen Gesundheitswesens erbringen, um die erforderlichen medizinischen Atteste zu auszustellen zur Bestätigung dessen, dass keine Krankheiten vorliegen, welche eine Gefahr für die Gesundheit der Bevölkerung darstellen (diese Atteste sind erforderlich, um einem Ausländer eine ständige Aufenthaltserlaubnis zu erteilen).

[...]

Quelle: Ministerstwo wnutrennich del Republik Belarus: Informazija dlja grashdan Ukrainy, pribywajuschtschich w Respubliku Belarus, <http://mvd.gov.by/ru/main.aspx?guid=208573>.

\section{Präsident Lukaschenka über die Rahmenbedingungen für ukrainische Flüchtlinge}

In Belarus halten sich derzeit 27.000 bis 30.000 Flüchtlinge aus der Ukraine auf, berichtete Aljaksandr Lukaschenka auf der Pressekonferenz für Journalisten russischer Regionalmedien am 17. Oktober in Minsk. »Und sie haben nicht die Absicht, zurückzukehren«, erklärte er.

Den Worten des Präsidenten zufolge, kommen aus der Ukraine »unterschiedliche Menschen«. In der Mehrzahl handelt es sich um »normale, verständnisvolle« Personen. Allerdings gäbe es auch diejenigen, die glauben, dass man sie »in Watte packen und auf Händen tragen« solle, "weil sie gelitten haben«. "Solche Personen ernüchtern wir. Wir verstehen, dass sie gelitten haben und sie es schwer haben, doch wir schaffen für sie alle erforderlichen Voraussetzungen, damit sie arbeiten und für sich und ihre Kinder den Lebensunterhalt verdienen können, so dass sie es nicht schlechter haben als in der Ukraine. Wir schaffen jedoch keine Rahmenbedingungen für sie, die ihnen einen Sonderstatus geben, denn dies könnte der Akzeptanz der Zugezogenen in unserer Bevölkerung schaden", so Lukschenka.

Seinen Worten zufolge haben die ukrainischen Flüchtlinge die Möglichkeit, in Belarus Arbeit zu finden, in vielen Fällen erhielten sie sogar eine Dienstwohnung. »Kommt hierher, wählt aus, arbeitet und verdient. Ja, in Minsk ist es sehr schwierig (Wohnraum zu erhalten, Anm. Nachrichtenagentur Belapan), doch in den Regionen ist es einfacher. 99,9\% verstehen dies, doch es gibt auch diejenigen, die in Minsk eine Wohnung wollen, wo sie dann sitzen und auf die Sozialhilfe warten werden«, kommentierte Lukaschenka.

Für die Neuankömmlinge aus der Ukraine, behauptet er, seien alle bürokratischen Hürden beim Grenzübertritt, beim Erhalt der Aufenthaltsgenehmigung und anderer Dokumente sowie der Arbeitsaufnahme beseitigt worden. Damit seien sie "faktisch den Belarussen gleichgestellt», betonte Lukaschenka.

„Wir haben 70.000 freie Stellen im Land, die Arbeitslosigkeit liegt bei 0,5\%. Es gibt weniger Arbeitslose als freie Stellen. Gesucht werden zu zwei Dritteln Arbeiter. Wir haben auch viele gute Stellen, doch diese verlangen eine sehr gute Ausbildung, nicht jeder Arbeitslose ist dafür geeignet. Gleichzeitig will nicht jeder Arbeitslose eine Arbeiterstelle annehmen. Es gibt freie Stellen in der Landwirtschaft, auch auf dem Bau gibt es nicht viele, doch einige freie Stellen. Allerdings sind hier auch die Anforderungen sehr hoch«, erläuterte das Staatsoberhaupt.

Quelle: W Belarusi nachoditsja do 30 tysjatsch beshenzew is Ukrainy, sajawljaet Lukaschenko, Nachrichtenagentur Belapan, 17.10.2014, <http://belapan.com/archive/2014/10/17/734171/>.

\section{Berichte ukrainischer Flüchtlinge}

$[\ldots]$

»Zu Beginn war es in der fremden Stadt sehr schwer. In der Verwaltung für Staatsbürgerschaft und Migration wurden wir vor die Tatsache gestellt, dass man uns, wenn wir binnen drei Monate keine Arbeit finden würden, aus dem Land nach Hause deportieren würde. Doch wohin nach Hause? Ich weiß nicht, ob es das noch gibt, unser Haus«, berichtet 
Olga. "Natürlich haben wir Arbeit gesucht. [Mein Mann] Denis hat auch fast sofort welche gefunden: Der Direktor der Eisenbetonfabrik ist ihm entgegengekommen und hat ihm gesagt, er suche begabte Jungs. Doch mich und meine Mutter wollte niemand haben. Die einen sagten, uns würden die erforderlichen Dokumente fehlen, die anderen vermissten den Aufenthaltstitel (den man nur durch die Aufnahme einer Beschäftigung erhält). In der Personalabteilung der Baumwollfabrik wurde uns offen gesagt, es sei für sie einfacher, Arbeiter aus Bangladesch einzustellen als Flüchtlinge aus der Ukraine. Nach dem Erscheinen des Präsidialerlasses wurde es allerdings etwas leichter, jetzt verspricht man mir eine Stelle im Kindergarten, doch meine Mutter will man bisher nicht einmal als Garderobenfrau einstellen.

Unsere Heimatstadt Suchodolsk ist fast achtmal kleiner als Baranowitschi. Sogar im Krieg war das Leben in der Ukraine billiger. Unser letztes Geld haben wir in Belarus für die Aufenthaltsgenehmigungen und für eine Wohnung fast ohne Möbel ausgegeben. Für Essen blieb uns keine `Kopeke`. Wir hatten auch keine warme Kleidung: wir waren in unseren Sommersachen gekommen.

Wir beschlossen, die Stadtbewohner um Hilfe zu bitten und veröffentlichten einen Brief auf einer Homepage mit Aufrufen. Am gleichen Tag riefen uns Städter an und boten Hilfe an. Sie schenkten uns einen Kühlschrank, halfen die Fenster zu verglasen. Jeden Tag kamen mehrere Menschen. Vor allem brachten sie Kleider. Wir haben das Notwendigste für uns genommen, alles andere haben wir an die Kirche weitergegeben, es gibt sicher noch andere vom Schicksal gebeutelte Menschen, die Hilfe brauchen.« [...]

Quelle: Kakshiwut beshenzy is Ukrainyw Baranowitschach, Intex-press, 27.9.2014, <http://www.intex-press.by/ru/news/society/17152/>.

\section{Internationale Flüchtlingshilfe}

Das Internationale Rote Kreuz (IRC) sowie die Internationale Rotkreuz- und Rothalbmond-Bewegung (IFRC) werden über 500.000 Schweizer Franken zur Unterstützung von ukrainischen Flüchtlingen in Belarus bereitstellen, berichtete der Generalsekretär des Belarussischen Roten Kreuzes Wiktar Kolbanau der Nachrichtenagentur BelTA.

Das IRC wird etwa 430.000 Schweizer Franken für ein sechsmonatiges Projekt bereitstellen. »Ich denke, wir werden dieses Geld bald erhalten«, sagte Wiktar Kolbanau. [...] Die IFRC wird etwa 130.000 Schweizer Franken für die Unterstützung von Ukrainern bereitstellen.

Des Weiteren plant das Schweizer Rote Kreuz, 200.000 Franken für die Bedarfe von ukrainischen Flüchtlingen zur Verfügung zu stellen. Weitere 20.000 Franken sollen als Extrareserve hinzukommen. Auch das Dänische und das Österreichische Rote Kreuz wollen helfen. Insgesamt haben wir etwa 900.000 Franken beantragt, um die Bedürfnisse der ukrainischen Flüchtlinge zu decken, so Wiktar Kalbanau. Personen aus der Ukraine können Voucher erhalten, um sich für einen bestimmten Betrag Essen, Kleider, Schuhe und Hygieneprodukte zu kaufen. Laut Wiktar Kolbanau haben sich bisher über 7.000 Ukrainer an das Belarussische Rote Kreuz gewandt.

[...]

Quelle: CRC, IFRC to allocate over CHF500k to help Ukrainian refugees in Belarus, BelTA, 5.12.2014, <http://eng.belta.by/all_news/ society/ICRC-IFRC-to-allocate-over-CHF500k-to-help-Ukrainian-refugees-in-Belarus_i_77874.html>.

\section{Verdienstmöglichkeiten für ukrainische Flüchtlinge}

$[\ldots]$

In der Datenbank des Arbeitsministeriums finden sich in jeder Region des Landes mehrere hundert Stellenangebote. Selbst in der Hauptstadt gibt es ausreichend freie Plätze. Die Firma »Elema» beispielsweise ist bereit 20 Näherinnen einzustellen. Sie bietet ein Gehalt von etwa 5 Mio. BYR und einen Wohnheimplatz. Die Traktorenwerke wollen Ukrainer mit mehreren Berufsprofilen einstellen. Besonders gefragt sind Schlosser für Montagearbeiten, hier werden mehr als 20 gesucht. Eine Wohnung wird nicht gestellt, doch das versprochene Gehalt reicht von 5,4 Mio. BYR für Transportfachkräfte bis zu 8,9 Mio. BYR für Gesenkschmiede. Die Minsker Fabrik für Heizanlagen braucht Dreher und Metallformer. Das Gehalt liegt zwischen 6 und 6,5 Mio. BYR.

Es ist auch ein Mangel an einheimischen Medizinern erkennbar. Die Belarussen arbeiten nicht gerne für das geringe Gehalt, das in Krankenhäusern und Polykliniken geboten wird. Daher werden nun Bürger aus der Ukraine eingestellt. In der 14. Zentralen Bezirkspolyklinik der Hauptstadt werden beispielsweise ein Chirurg, ein Gynäkologe, ein Endokrinologe und Krankenschwestern gesucht. Den Krankenschwestern werden 3,5 Mio. BYR geboten, den Ärzten 
eine Million mehr. In der 4. Städtischen Klinik namens N.E. Sawtschenko werden bis zu 7 Mio. BYR Lohn geboten, dafür erhalten hier Reinigungskräfte nur 2 Mio. BYR, ohne dass Wohnraum geboten wird.

In den Kindergärten gibt es ebenfalls genügend freie Stellen. Eine Erziehungshelferin erhält 3 Mio. BYR, eine Küchenhilfe 2,8 Mio. BYR. Ein Erzieher kann nur mit wenig mehr rechnen: etwa 3,5 Mio. BYR.

Verkäuferinnen können 3,5 bis 5 Mio. BYR verdienen. Auf dem Bau wird in der Hauptstadt mehr gezahlt. Schweißern, Montagearbeitern, Vorarbeitern werden 8 bis 10 Mio. BYR versprochen.

[...]

In den Regionen gibt es ebenfalls eine beindruckende Liste an freien Stellen für Bürger aus der Ukraine. Es werden Arbeiter, Bauarbeiter, Verkäuferinnen, medizinisches Personal und Näherinnen gesucht. Sehr viele freie Stellen gibt es in der Landwirtschaft. Ob die Migranten allerdings das Personalproblem auf dem Land lösen können angesichts der dort gebotenen Löhne?

Die wenigen finanziell attraktiven Angebote fallen sofort auf. Im Landwirtschaftlichen Kombinat »Heiliger Wille» im Bezirk Iwazewitschi werden ein Chefingenieur, ein Veterinär und ein Tierpfleger für jeweils 8 Mio. BYR gesucht. Darüber hinaus wird sogar noch Wohnraum angeboten.

[...]

Im Internet finden sich auch zahlreiche Stellengesuche von Bürgern aus dem Nachbarland. Es gibt Gesuche, in denen die Leute "praktisch zu jeder Arbeit für jedes Gehalt bereit» sind. Doch nicht alle ukrainischen Bürger wollen für einen Lohn von 300-400 US-Dollar arbeiten. Beispielsweise ist ein Fahrer mit Führerschein der Klasse B und C aus dem Gebiet Luhansk für 800 US-Dollar im Monat zu Fahrten bereit. Ein Mechanikingenieur aus Donezk will eine Arbeit für mindesten 1.000 US-Dollar finden. Ein Verkaufsmanager aus Charkiw sucht eine Stelle mit 700 US-Dollar Gehalt. Und Witalij aus Odessa sucht Arbeit als Gabelstaplerfahrer für 1.000 US-Dollar im Monat. $\{\ldots]$

Quelle: Skolko w Belarusi gotowy platit migrantam is Ukrainy? Belaruski tschas, 19.11.2014, <http://zautra.by/art.php?\&sn_nid=16840>.

\section{Chance für die demographische Entwicklung}

Der migrationsbedingte Bevölkerungszuwachs in Belarus betrug im Januar - September 2014 insgesamt 8.474 Personen. Dies sind 605 Personen mehr als im Vorjahreszeitraum. [... Insgesamt] stieg die Einwohnerzahl von Belarus um 6.929 Personen und belief sich dem nationalen Statistikamt zufolge zum 1. Oktober auf 9.475.100 Personen.

[...] Die demographische Entwicklung ist folglich derart, dass wir unbedingt Migranten brauchen. Denn die Experten bestätigen, dass die Bevölkerung von Belarus sich verringern wird. [...] Das Staatsprogramm für demographische Sicherheit für 2011-2015 sieht daher eine Zuwanderung von 60.000 Personen innerhalb von fünf Jahren vor. Für Belarus wäre es am besten, wenn Personen aus einem Land mit ähnlicher Kultur und Mentalität zuwandern und sich zum Arbeiten niederlassen würden, erläuterte Andrej Elisejeeu vom Belarussischen Institut für strategische Studien (BISS) in seinem Kommentar für Nawiny.by.

Er betonte, dass sich für Belarus infolge des Kriegs in der Ukraine eine einzigartige Situation ergeben habe: "Die Folgen des Kriegs im Donbass sind schrecklich. Belarus muss der Zivilbevölkerung Hilfe leisten und dabei seine nationalen Interessen berücksichtigen. Die Vereinfachung der Verfahren für ukrainische Staatsbürger zum Erhalt von Staatshilfen ist daher aktuell und richtig."

\{...] Wichtig ist es zu verstehen, dass die Zuwanderung aus der Ukraine nach Belarus ein vorübergehendes Phänomen ist und unser Land diesen Augenblick nutzen sollte.

„Dies entspricht den nationalen Interessen von Belarus. Zudem kann unser Land in diesem Falle eine humanitäre Rolle spielen, was ebenfalls sehr wichtig ist«, unterstrich Andrej Elisejeeu.

Quelle: Ukrainskich beshenzew belorusskaja statistika ne widit, Nawiny.by, 29.10.2014, <http://naviny.by/rubrics/society/2014/10/29/ ic_articles_116_187413/>. 


\section{Oktober bis 10. Dezember 2014}

\begin{tabular}{|c|c|}
\hline 01.10 .2014 & $\begin{array}{l}\text { Die Stadt Minsk erteilt Smizer Wajtjuschkewitsch eine Auftrittsgenehmigung. Der belarussische Sänger und } \\
\text { Komponist darf erstmals seit März } 2011 \text { wieder offiziell ein Konzert in Belarus geben. Wajtjuschkewitsch } \\
\text { stand bis dahin auf einer »schwarzen Liste« von Personen, deren Lieder in Belarus nicht offiziell gespielt wer- } \\
\text { den durften. }\end{array}$ \\
\hline 01.10 .2014 & $\begin{array}{l}\text { Aljaksandr Lukaschenka erklärt sich in einem Interview mit dem Fernsehsender »Euronews« dazu bereit, } \\
\text { belarussische Soldaten als Friedensmission in die Ukraine zu schicken, um zur Regulierung des Konflikts in } \\
\text { der Ostukraine beizutragen. Des Weiteren betont Lukaschenka, dass Belarus nicht plane, die selbst ernann- } \\
\text { ten Donezker und Luhansker Volksrepubliken anzuerkennen, und er weiterhin für den Erhalt der territoria- } \\
\text { len Integrität der Ukraine eintrete. }\end{array}$ \\
\hline 01.-02.10.2014 & $\begin{array}{l}\text { Der stellvertretende belarussische Ministerpräsident Michail Rusy und der polnische Wirtschaftsminister } \\
\text { Janusz Piechociński nehmen am 18. belarussisch-polnischen Wirtschaftsforum in Brest teil. Diskutiert wer- } \\
\text { den u. a. Perspektiven der bilateralen Kooperation in den Bereichen Logistik und Handel sowie im Bank- } \\
\text { und Industriesektor. }\end{array}$ \\
\hline 02.10 .2014 & $\begin{array}{l}\text { Die Organisatoren der Initiative »Volksreferendum» übergeben dem Parlament einen von } 50.000 \text { belarussi- } \\
\text { schen Bürgern unterschriebenen Aufruf, in der sie die Durchführung einer »Volksabstimmung«mit insgesamt } \\
\text { sechs Fragen verlangen. Hierzu gehört u. a. die Begrenzung der Amtszeit des Präsidenten, die Direktwahl von } \\
\text { Bürgermeistern und Gouverneuren, die Annäherung an die EU sowie staatliche Entschädigungszahlen an } \\
\text { die Bevölkerung für die Inflationsverluste des Jahres 2014. Zu den Initiatoren des "Volksreferendums« gehö- } \\
\text { ren u. a. die Bürgerinitiative "Sag die Wahrheit» von Uladsimir Njakljaeu, die Bewegung "Für die Freiheit» } \\
\text { von Aljaksandr Milinkewitsch und weitere Oppositionskräfte. }\end{array}$ \\
\hline 03.-05.10.2014 & $\begin{array}{l}\text { Eine belarussische Parlamentsdelegation unter Leitung des stellvertretenden Parlamentsvorsitzenden Wiktar } \\
\text { Huminski nimmt an der Herbsttagung der Parlamentarischen Versammlung der OSZE in Genf teil. }\end{array}$ \\
\hline 05.-10.10.2014 & $\begin{array}{l}\text { Im Rahmen der zweiten Woche gegen die Todesstrafe organisieren belarussische Menschenrechtler landesweit } \\
\text { mehrere Diskussionen, Aktionen, Ausstellungen und Filmvorführungen. Belarus ist weiterhin der einzige Staat } \\
\text { in Europa, der die Todesstrafe anwendet. } 2014 \text { kam die Todesstrafe in Belarus bisher zweimal zur Anwendung. }\end{array}$ \\
\hline 06.10 .2014 & $\begin{array}{l}\text { Der Homeler Aktivist Jury Rubzou wird von einem Minsker Gericht der »Beleidigung des Richters «ür schul- } \\
\text { dig befunden und zu 1,5 Jahren Haft im offenen Vollzug verurteilt. Rubzou war bereits Ende April } 2014 \text { zu } \\
\text { einer 25tägigen Haftstrafe verurteilt worden, da er beim traditionellen Tschernobyl-Marsch der Opposition } \\
\text { zum 26. April ein T-Shirt mit der Aufschrift »Lukaschenka hau ab!" getragen hatte. }\end{array}$ \\
\hline 07.10 .2014 & $\begin{array}{l}\text { Ministerpräsident Michail Mjasnikowitsch und sein russischer Amtskollege Dmitrij Medwedew einigen sich in } \\
\text { Sotschi über die künftigen Zollsätze für die in Belarus produzierten Erdölprodukte. Auslöser war das in Russ- } \\
\text { land im August } 2014 \text { beschlossene sogenannte »Steuermanöver«, das eine Senkung der Ausfuhrzölle für Erdöl } \\
\text { und Erdölprodukte und gleichzeitig eine innerrussische Steuererhöhung für die Rohstoffgewinnung vorsieht. }\end{array}$ \\
\hline 07.10 .2014 & $\begin{array}{l}\text { Der freie Journalist Andrej Mjaleschka wird von einem Hrodnauer Gericht wegen »illegaler Herstellung von } \\
\text { Medienprodukten« für seine Kooperation mit dem in Belarus nicht-akkreditierten polnischen »Radio Razija» } \\
\text { zu einer Geldstrafe in Höhe von über } 5 \text { Millionen BYR verurteilt. Einen Tag später wird der Journalist Aljak- } \\
\text { sandr Burakou unter dem gleichen Vorwurf für seine Zusammenarbeit mit der »Deutschen Welle« ebenfalls } \\
\text { zu einer Geldstrafe verurteilt. }\end{array}$ \\
\hline 08.10 .2014 & $\begin{array}{l}\text { Die OSZE-Beauftragte für die Freiheit der Medien Dunja Mijatović ruft die belarussischen Behörden dazu } \\
\text { auf, freie Journalisten nicht länger für ihre Kooperation mit in Belarus nicht-akkreditierten ausländischen } \\
\text { Medien zu verfolgen. }\end{array}$ \\
\hline 09.10 .2014 & $\begin{array}{l}\text { Der Vertrag über die Eurasische Wirtschaftsunion (EAWU) wird durch die beiden Kammern der belarussi- } \\
\text { schen Nationalversammlung ratifiziert. In einer zusätzlichen Erklärung betont das Parlament zugleich, dass } \\
\text { die belarussische Seite nur unter der Voraussetzung zur Einhaltung der vertraglichen Bestimmungen bereit } \\
\text { sei, solange die anderen Vertragspartner diesen gleichfalls folgen würden. }\end{array}$ \\
\hline 09.10 .2014 & $\begin{array}{l}\text { Im Vorfeld des Internationalen Tages gegen die Todesstrafe am 10. Oktober fordern die EU und der Euro- } \\
\text { parat in einer gemeinsamen Erklärung die belarussischen Behörden dazu auf, ein Moratorium für die Todes- } \\
\text { strafe einzuführen. }\end{array}$ \\
\hline
\end{tabular}




\begin{tabular}{|c|c|}
\hline 09.10 .2014 & $\begin{array}{l}\text { Beim Fußballspiel zwischen Belarus und der Ukraine in Barisau skandieren zahlreiche Fans politische Losungen } \\
\text { und tragen nationale Symbole. Die belarussischen Sicherheitskräfte hatten bereits im Vorfeld etliche ukraini- } \\
\text { sche Fans an der Einreise nach Belarus gehindert. Nach dem Spiel kommt es zu Verhaftungen und am Folgetag } \\
\text { werden } 24 \text { belarussische und ukrainische Fußballfans zu kurzen Haft- bzw. Geldstrafen verurteilt. Die ukrai- } \\
\text { nischen Fußballfans werden jedoch nach einer Intervention des ukrainischen Konsuls auf freien Fuß gesetzt. }\end{array}$ \\
\hline 10.10 .2014 & $\begin{array}{l}\text { In Minsk findet eine Ratssitzung der GUS-Staatschefs statt. Bei der anschließenden Sitzung des Obersten } \\
\text { Eurasischen Wirtschaftsrats unterzeichnen die Staatschefs von Armenien, Belarus, Russland und Kasachs- } \\
\text { tan den Vertrag über den Beitritt Armeniens zur Eurasischen Wirtschaftsunion (EAWU). Armenien wird } \\
\text { damit bereits zum 1. Januar 2015, wenn der EAWU-Vertrag in Kraft tritt, vollwertiges Mitglied. Die Staats- } \\
\text { chefs vereinbaren zugleich eine Roadmap für den Beitritt von Kyrgistan zur EAWU. Ebenso beschließen sie } \\
\text { die Auflösung der Eurasischen Wirtschaftsgemeinschaft, an deren Stelle die EAWU tritt. }\end{array}$ \\
\hline 13.10.2014 & $\begin{array}{l}\text { Der polnische Abgeordnete Bogdan Andrzej Zdrojewski wird zum Leiter der Delegation für die Beziehun- } \\
\text { gen zu Belarus im Europäischen Parlament gewählt. }\end{array}$ \\
\hline 14. -15.10 .2014 & $\begin{array}{l}\text { Eine parlamentarische Delegation unter Leitung des Vorsitzenden des Rats der Republik Anatol Rubinau } \\
\text { hält sich zu einem Arbeitsbesuch in der Türkei auf. Rubinau wird auch vom türkischen Staatschef Erdogan } \\
\text { empfangen. }\end{array}$ \\
\hline 16.10 .2014 & $\begin{array}{l}\text { Der Oberkommandeur der russischen Luftwaffe Viktor Bondarew kündigt an, dass } 2016 \text { in Babrujsk ein wei- } \\
\text { terer russischer Luftwaffenstützpunkt mit SU-27-Kampfjets eröffnet werden soll. Derzeit sind bereits russi- } \\
\text { sche Kampfjets in Baranowitschi stationiert. }\end{array}$ \\
\hline 17.10 .2014 & $\begin{array}{l}\text { Aljaksandr Lukaschenka erklärt während einer Pressekonferenz für russische Journalisten, die Ukraine habe } \\
\text { den Anschluss der Krim an Russland durch ihre stark antirussische Politik nach der Revolution selbst pro- } \\
\text { voziert. Gleichzeitig betont der Präsident, es sei inakzeptabel, wenn ein Staat gegen die territoriale Integri- } \\
\text { tät eines anderen verstoße. }\end{array}$ \\
\hline 20.-23.10.2014 & $\begin{array}{l}\text { Eine EU-Delegation führt in Minsk Konsultationen zu Umweltfragen mit Vertretern des Außen- und des } \\
\text { Umweltministeriums sowie internationaler Organisationen und NROs durch. Der Besuch der EU-Delega- } \\
\text { tion dient der Vorbereitung des Kooperationsprogramms zwischen der EU und Belarus für das Jahr } 2015 \text {. }\end{array}$ \\
\hline 23.-24.10.2014 & $\begin{array}{l}\text { Eine iranische Delegation unter Leitung des stellvertretenden Außenministers Ebrahim Rahimpur hält sich } \\
\text { zu Konsultationen in Minsk auf und trifft sich u. a. mit Außenminister Makej. Zu den Gesprächsthemen } \\
\text { gehören gemeinsame Projekte in Investitions-, Wirtschafts- und Handelsbereichen. }\end{array}$ \\
\hline 24.10 .2014 & $\begin{array}{l}\text { Nach Angaben des Pressedienstes der Bewegung "Für die Freiheit« verweigern die Minsker Behörden die } \\
\text { Genehmigung für } 20 \text { Kundgebungen vor der russischen Botschaft, bei denen zur Einhaltung der Waffen- } \\
\text { ruhe in der Ukraine aufgerufen werden sollte. }\end{array}$ \\
\hline 24.10 .2014 & $\begin{array}{l}\text { Der UN-Menschenrechtsausschuss erklärt, dass die belarussischen Behörden mit der gegen demokratische } \\
\text { Prinzipien verstoßenden mehrmaligen Weigerung, die Menschenrechtsorganisation "Wjasna« zu registrieren, } \\
\text { deren Leiter Ales Bjaljazki faktisch gezwungen haben, die Aktivitäten von „Wjasna« unter Verletzung des gel- } \\
\text { tenden belarussischen Rechts zu organisieren. Da die belarussischen Behörden damit Bjaljazki und andere } \\
\text { Mitglieder von „Wjasna« an der Ausübung ihrer Grundrechte gehindert haben, sei die am } 24 \text {. Oktober } 2011 \\
\text { erfolgte Verurteilung von Bjaljazki zu einer mehrjährigen Haftstrafe nicht mit internationalem Recht verein- } \\
\text { bar. Der Ausschuss fordert daher die belarussischen Behörden auf, den im Juni } 2014 \text { vorzeitig aus der Haft } \\
\text { entlassenen Bjaljazki vollständig zu rehabilitieren und Entschädigung zu leisten, das Menschenrechtszen- } \\
\text { trum »Wjasna« offiziell zu registrieren und die NGO-Gesetzgebung an internationale Standards anzupassen. }\end{array}$ \\
\hline 25.-29.10.2014 & $\begin{array}{l}\text { Aljaksandr Lukaschenka hält sich zu einem Arbeitsbesuch in den Vereinigten Arabischen Emiraten auf und trifft } \\
\text { sich u. a. mit dem Kronprinz von Abu Dhabi Scheich Mohammed bin Zayed Al Nahyan. Offiziellen Medien- } \\
\text { berichten zufolge werden eine Reihe von Absprachen über gemeinsame Projekte in Wirtschafts-, Investitions- } \\
\text { und Wissenschaftsbereichen abgeschlossen. In den unabhängigen Medien wird der mehrtägige Aufenthalt } \\
\text { des Präsidenten, bei dem auch ein Eishockeyspiel auf dem Programm stand, jedoch als Kurzurlaub bewertet. }\end{array}$ \\
\hline 28.10.2014 & $\begin{array}{l}\text { Der Sonderberichterstatter für Belarus Miklós Haraszti präsentiert der 69. UN-Generalversammlung in New } \\
\text { York seinen neuen Bericht zur Lage der Menschenrechtsituation. Demzufolge hat sich die Menschenrechts- } \\
\text { situation in Belarus im laufenden Jahr nicht wesentlich verändert. }\end{array}$ \\
\hline 28.10.2014 & $\begin{array}{l}\text { Das belarussische Außenministerium erklärt anlässlich der am 26. Oktober erfolgten Parlamentswahlen in } \\
\text { der Ukraine, Belarus respektiere die Wahl des ukrainischen Volkes. }\end{array}$ \\
\hline
\end{tabular}




\begin{tabular}{|c|c|}
\hline 28.10 .2014 & $\begin{array}{l}\text { In Genf finden Konsultationen über den Beitritt von Belarus zur Welthandelsorganisation (WTO) statt. Auf } \\
\text { belarussischer Seite werden die Gespräche vom stellvertretenden Außenminister Aljaksandr Hurjanau und } \\
\text { auf WTO-Seite vom Vorsitzenden der Belarus-Arbeitsgruppe Mehmet Haluk Ilicak sowie Vertretern des } \\
\text { WTO-Sekretariats geleitet. }\end{array}$ \\
\hline 30.10 .2014 & $\begin{array}{l}\text { Der Europäische Rat verlängert die EU-Sanktionen gegen Belarus um ein weiteres Jahr, da das offizielle Minsk } \\
\text { nicht die erforderlichen Voraussetzungen für ihre Aufhebung geschaffen habe. Es erfolgen jedoch einige Ver- } \\
\text { änderungen an der Sanktionsliste. Demnach erstrecken sich die Einreiseverbote nun auf } 201 \text { Personen (bis- } \\
\text { her 225), zudem gelten für } 17 \text { Unternehmen (bisher 25) eine Sperrung der Vermögenswerte und Handelsres- } \\
\text { triktionen. Das belarussische Außenministerium bezeichnet den Beschluss als eine verpasste Möglichkeit, } \\
\text { das Haupthindernis zur Normalisierung der beidseitigen Beziehungen zu beseitigen. Es sieht jedoch in der } \\
\text { Reduzierung der Sanktionen einen Schritt in die richtige Richtung. }\end{array}$ \\
\hline 31.10 .2014 & $\begin{array}{l}\text { Der Aktivist der Bewegung »Für die Freiheit« Uladsislau Koschaleu wird von einem Minsker Gericht wegen } \\
\text { "Verletzung der Organisations- und Durchführungsordnung von Massenveranstaltungen« zu } 3 \text { Tagen Haft } \\
\text { verurteilt. Koschaleu war am 30. Oktober } 2014 \text { festgenommen worden, nachdem er Unterschriften für die } \\
\text { Initiative »Volksabstimmung« in einem studentischen Wohnheim gesammelt hatte. }\end{array}$ \\
\hline 01.-05.11.2014 & $\begin{array}{l}\text { Eine Delegation unter Leitung des Oberbefehlshabers der Streitkräfte von Myanmar Min Aung Hlaing hält } \\
\text { sich zu einem Arbeitsbesuch in Minsk auf und trifft sich u. a. mit Präsident Aljaksandr Lukaschenka und } \\
\text { Ministerpräsident Michail Mjasnikowitsch. Die Gastgeber betonen das Interesse von Belarus an einer Koope- } \\
\text { ration mit Myanmar im militärischen Bereich sowie in den Bereichen Gesundheitswesen, Landwirtschaft } \\
\text { und Technologietransfer. }\end{array}$ \\
\hline 02.11 .2014 & $\begin{array}{l}\text { Anlässlich des Totengedenktags "Dsjady» organisiert die Belarussische Konservative Partei BNF mit Geneh- } \\
\text { migung der Stadt Minsk einen Gedenkzug, der mit einer Kundgebung in Kurapaty endet. In } 1988 \text { hatte der } \\
\text { BNF-Gründer Sjanon Pasnjak erstmals die stalinistischen Massenerschießungen, die während der 1930er } \\
\text { Jahre in Kurapaty stattfanden, öffentlich gemacht. Seitdem finden jährlich zum 2. November Veranstaltun- } \\
\text { gen in Kurapaty statt. }\end{array}$ \\
\hline 04.11.2014 & $\begin{array}{l}\text { Die belarussischen Behörden geben die Hinrichtung von Aljaksandr Hrunou, der im April } 2014 \text { wegen Mord } \\
\text { durch das Oberste Gericht von Belarus zum Tode verurteilt worden war, bekannt. Die EU, der Europarat } \\
\text { und weitere internationale Organisationen verurteilen in den folgenden Tagen die dritte Vollstreckung der } \\
\text { Todesstrafe in Belarus im laufenden Jahr. Sie fordern die belarussischen Behörden erneut dazu auf, ein Mora- } \\
\text { torium für die Todesstrafe einzuführen. }\end{array}$ \\
\hline 04.-05.11.2014 & $\begin{array}{l}\text { Der Direktor des Amtes für Osteuropa im US-Außenministerium Alexander Kasanof hält sich in Minsk zu } \\
\text { einem Arbeitsbesuch auf. Zu seinen Gesprächspartnern gehören der Abteilungsleiter für Amerika im bela- } \\
\text { russischen Außenministerium sowie Vertreter der belarussischen Opposition. }\end{array}$ \\
\hline 05.11.2014 & $\begin{array}{l}\text { Die Behörde für Staatsbürgerschaft und Migration des Perschamajsker Stadtbezirks in Minsk entscheidet, } \\
\text { dass die Menschenrechtlerin Aljona Tankatschowa, die als russische Staatsbürgerin seit Jahrzehnten in Bela- } \\
\text { rus lebt, innerhalb von } 30 \text { Tagen das Land zu verlassen habe und es für drei Jahre nicht mehr betreten dürfe. } \\
\text { Der Beschluss wird damit begründet, dass Tankatschowa aufgrund mehrmaliger Geschwindigkeitsüber- } \\
\text { schreitungen ihres PKWs eine Gefahr für die öffentliche Ordnung darstelle. Tankatschowa legt gegen die } \\
\text { Entscheidung Berufung ein. }\end{array}$ \\
\hline 06.11 .2014 & $\begin{array}{l}\text { Präsident Lukaschenka ernennt den bisherigen Minister für Wohnungs- und Kommunalwirtschaft Andrej } \\
\text { Schorez zum neuen Minsker Oberbürgermeister. Neuer Finanzminister wird der bisherige erste stellvertre- } \\
\text { tende Finanzminister Uladsimir Amarin. }\end{array}$ \\
\hline 12.11.2014 & $\begin{array}{l}\text { Die Vorsitzende der Zentralen Wahlkommission Lydija Jarmoschyna nennt bei einer Konferenz in Minsk den } \\
\text { 15. November } 2015 \text { als das wahrscheinlichste Datum für die nächsten Präsidentschaftswahlen. }\end{array}$ \\
\hline 12.-13.11.2014 & $\begin{array}{l}\text { Eine internationale UNICEF-Konferenz zum Thema Kinderschutz, an der Delegationen aus } 21 \text { Staaten teil- } \\
\text { nehmen, findet auf belarussische Initiative in Minsk statt. Der UNICEF-Regionaldirektorin für Mittel- und } \\
\text { Osteuropa und die GUS Marie-Pierre Poirier zufolge verfügt Belarus über ausreichende Erfahrung in der } \\
\text { Erhebung von Daten über Gewalt gegen Kinder, welche andere Staaten an ihre länderspezifischen Bedin- } \\
\text { gungen anpassen könnten. }\end{array}$ \\
\hline 13.-14.11.2014 & $\begin{array}{l}\text { Das Informationszentrum des Europarates in Minsk führt gemeinsam mit der NRO »Penal Reform Inter- } \\
\text { national« sowie mehreren belarussischen NRO eine internationale Konferenz gegen die Todesstrafe durch. } \\
\text { An der Konferenz beteiligen sich Experten, Regierungs- und NRO-Vertreter aus Belarus, Polen, Russland } \\
\text { und der Ukraine. }\end{array}$ \\
\hline
\end{tabular}




\begin{tabular}{|c|c|}
\hline 14.11.2014 & $\begin{array}{l}\text { In London findet das belarussisch-britische Investitionsforum statt, und in Wien führt die Österreichische } \\
\text { Wirtschaftskammer in Kooperation mit der Belarussischen und Russischen Wirtschaftskammer das Wirt- } \\
\text { schaftsforum Österreich-Belarus-Russland durch. Beim Forum in Wien wird eine Vereinbarung über die Ein- } \\
\text { richtung eines Belarussisch-Österreichischen Wirtschaftsrats unterzeichnet. }\end{array}$ \\
\hline 17.11.2014 & $\begin{array}{l}\text { Der stellvertretende Vorsitzende der konservativ-christlichen Partei BNF Jury Beljanki wird wegen des Verstoßes } \\
\text { gegen das Gesetz über Massenveranstaltungen bei der Kundgebung anlässlich des Totengedenktags "Dsjady« } \\
\text { am } 2 \text {. November von einem Minsker Gericht zu einer Geldstrafe in Höhe von } 3 \text { Millionen Rubel verurteilt. }\end{array}$ \\
\hline 18.11.2014 & $\begin{array}{l}\text { Die Außenministerien von Belarus und Russland kommen zu einer gemeinsamen Sitzung in Minsk zusam- } \\
\text { men, um sich u. a. über die Krise in der Ukraine, die EU-Sanktionen gegen beide Staaten sowie die Statio- } \\
\text { nierung von US-amerikanischen und europäischen Raketenabwehrsystemen in Europa auszutauschen. Dabei } \\
\text { ruft Russlands Außenminister Sergej Lawrow zur Wiederaufnahme des Ukraine-Dialogs im Minsker For- } \\
\text { mat auf. Der belarussische Außenminister Uladsimir Makej spricht sich u. a. für eine engere Einbeziehung } \\
\text { Russlands in die "Östliche Partnerschaft" aus. }\end{array}$ \\
\hline 18.-19.11.2014 & $\begin{array}{l}\text { Der Leiter des Departements für Russland, Östliche Partnerschaft, Zentralasien, regionale Zusammenar- } \\
\text { beit und OSZE beim Europäischen Auswärtigen Dienst Gunnar Wiegand hält sich in Minsk auf, um an der } \\
\text { 4. Konsultationsrunde zwischen Belarus und der EU zu Modernisierungsfragen teilzunehmen. Zu seinen } \\
\text { Gesprächspartnern gehören u. a. Außenminister Uladsimir Makej, dessen Stellvertreterin Aljona Kuptschyna } \\
\text { sowie Vertreter der belarussischen Zivilgesellschaft. }\end{array}$ \\
\hline 21.11.2014 & $\begin{array}{l}\text { Der Aufsichtsrat der russischen Landwirtschaft erklärt, dass über } 10 \% \text { des deklarierten Reexports von EU- } \\
\text { Lebensmitteln aus Belarus nach Kasachstan de facto in Russland versanden. U. a. wurden über } 11.000 \mathrm{t} \\
\text { Obst und Gemüse aus der EU sowie etwa } 300 \text { t EU-Fleisch mit deklarierter brasilianischer Herkunft auf- } \\
\text { gedeckt. Russland schränkt daraufhin ab dem } 24 \text {. November den Transit belarussischer Waren über russi- } \\
\text { sches Gebiet drastisch ein. }\end{array}$ \\
\hline 23.11.2014 & $\begin{array}{l}\text { Das belarussische Wirtschaftsministerium legt den Entwurf der »Nationalen Strategie für nachhaltige soziale } \\
\text { und wirtschaftliche Entwicklung«, die bis } 2030 \text { umgesetzt werden soll, zur öffentlichen Diskussion vor. Die } \\
\text { Strategie wurde in Zusammenarbeit mit Forschungsinstitutionen, Wirtschaftsakteuren, der UN-Vertretung, der } \\
\text { Internationalen Bildungs- und Begegnungstätte "Johannes Rau« Minsk sowie weiteren Akteuren vorbereitet. }\end{array}$ \\
\hline 24.-25.11.2014 & $\begin{array}{l}\text { Eine belarussische Delegation unter Führung des Leiters der Konsularabteilung des Außenministeriums Ihar } \\
\text { Fissenka nimmt in Brüssel an der zweiten Runde der Konsultationen über die Visaerleichterungs- und Rück- } \\
\text { übernahmeabkommen zwischen Belarus und der EU teil. }\end{array}$ \\
\hline 25.11.2014 & $\begin{array}{l}\text { Der Aktivist der Partei »Belarussische Christliche Demokratie« Andrej Haidukou wird wegen »Organisation } \\
\text { und Durchführung einer nicht sanktionierten Massenaktion« von einem Polazker Gericht zu } 10 \text { Tagen Haft } \\
\text { verurteilt. Haidukou war am 21. November in Nawapolazk und am 24. November in Polazk festgenommen } \\
\text { worden, als er Flyer über die politischen Gefangenen verteilt hatte. }\end{array}$ \\
\hline 26.-27.11.2014 & $\begin{array}{l}\text { Der Generalsekretär des Zentralkomitees der Kommunistischen Partei Vietnams Nguyễn Phú Trọng hält sich zu } \\
\text { einem Arbeitsbesuch in Minsk auf. Aljaksandr Lukaschenka kündigt während des Treffens mit dem Generalse- } \\
\text { kretär an, er sehe Vietnam als Ausgangspunkt für das wirtschaftliche Engagement von Belarus in Südostasien. }\end{array}$ \\
\hline 28.11.2014 & $\begin{array}{l}\text { Die Abschiebung der Menschenrechtsaktivistin Aljona Tankatschowa wird von einem Minsker Gericht um } \\
\text { einen Monat verschoben. }\end{array}$ \\
\hline 01.12 .2014 & In Warschau findet ein belarussisch-polnisches Investitionsforum statt. \\
\hline 01.-02.12.2014 & $\begin{array}{l}\text { In Vilnius (01.12.) und Minsk (02.12) findet eine durch die deutsch-belarussische Gesellschaft organisierte } \\
\text { internationale Konferenz zum Thema »Herausforderungen und Chancen für die Beziehungen zwischen Bela- } \\
\text { rus und der EU«statt. An der Konferenz nehmen Diplomaten und Politiker aus Belarus und EU-Staaten sowie } \\
\text { zahlreiche Experten teil. Im Ergebnis wird eine Reihe von Politikempfehlungen erarbeitet. }\end{array}$ \\
\hline 02.12 .2014 & $\begin{array}{l}\text { Der freie Journalist Andrej Mjaleschka wird erneut von einem Hrodnauer Gericht wegen »illegaler Herstel- } \\
\text { lung von Medienprodukten« für seine Kooperation mit dem in Belarus nicht-akkreditierten polnischen »Radio } \\
\text { Razija» zu einer Geldstrafe verurteilt. }\end{array}$ \\
\hline 03.12 .2014 & $\begin{array}{l}\text { Präsident Lukaschenka nimmt zu den russischen Vorwürfen, dass Belarus illegal verbotene EU-Waren nach } \\
\text { Russland einführe, Stellung. Er erklärt, dass der Transit von Waren für die belarussische Wirtschaft von ent- } \\
\text { scheidender Bedeutung sei. Sein Land würde sich daher an internationale Rechtsnormen halten und den Tran- } \\
\text { sit nicht einschränken. Russland müsse selber für die Einhaltung der beschlossenen Einfuhrverbote sorgen. }\end{array}$ \\
\hline 04.12 .2014 & $\begin{array}{l}\text { Belarussische Oppositionsvertreter besprechen in Warschau mit polnischen Abgeordneten die Lage in Bela- } \\
\text { rus im Vorfeld der Präsidentschaftswahlen } 2015 .\end{array}$ \\
\hline
\end{tabular}




\begin{tabular}{|l|l|}
\hline 04.-05.12.2014 & $\begin{array}{l}\text { Außenminister Uladsimir Makej erklärt während der Plenarsitzung des OSZE-Außenministerrates im schwei- } \\
\text { zerischen Basel, strukturelle Probleme im Sicherheitsbereich in der OSZE-Region seien seit der Krise in der } \\
\text { Ukraine noch offensichtlicher geworden. Makej trifft sich im Laufe der Sitzung u. a. mit dem Generalsekretär } \\
\text { der OSZE Lamberto Zannier, dem Direktor der OSZE-Behörde für demokratische Institutionen und Men- } \\
\text { schenrechte Michael Link und dem Generalsekretär des Europarates Thorbjørn Jagland. }\end{array}$ \\
\hline 05.12.2014 & $\begin{array}{l}\text { Der 2013 zu einem Jahr Haft verurteilte oppositionelle Aktivist Wassil Parfjankou, der von der EU als poli- } \\
\text { tischer Gefangener betrachtet wird, wird aus der Haft entlassen. }\end{array}$ \\
\hline 08.12.2014 & $\begin{array}{l}\text { Eine belarussische Delegation nimmt an der Konferenz »Doing Business mit der Eurasischen Wirtschafts- } \\
\text { union« in Washington teil. Hauptthemen der Konferenz sind Fragen der Zusammenarbeit zwischen den USA } \\
\text { und den eurasischen Staaten im Wirtschafts- und Investitionsbereich. }\end{array}$ \\
\hline 09.12.2014 & $\begin{array}{l}\text { Die stellvertretende Außenministerin Aljona Kuptschyna kündigt in einem Interview mit »Radio Belarus« } \\
\text { an, die Herstellung und der Ausbau freundschaftlicher Beziehungen zu EU-Staaten sei nach wie vor eine der } \\
\text { Prioritäten der belarussischen Außenpolitik. Kuptschyna weist auf positive Veränderungen in den Beziehun- } \\
\text { gen zwischen Belarus und der EU hin und äußert die Hoffnung, dass die EU-Sanktionen gegen Belarus auf- } \\
\text { gehoben werden. }\end{array}$ \\
\hline 10.12.2014 & $\begin{array}{l}\text { In mehreren belarussischen Städten finden Veranstaltungen und Kundgebungen zum Internationalen Tag } \\
\text { der Menschenrechte statt. }\end{array}$ \\
\hline
\end{tabular}

Zusammengestellt auf der Grundlage der Meldungen der Nachrichten-Agentur BelaPAN und der Homepage naviny.by.

Sie können die gesamte Chronik ab dem 14.03.2011 auch auf<http://www.laender-analysen.de/belarus/> unter dem Link »Chronik«lesen.

Mit finanzieller Unterstützung des Bundesministeriums für wirtschaftliche Zusammenarbeit und Entwicklung (BMZ)

Herausgeber: Forschungsstelle Osteuropa an der Universität Bremen, Deutsche Gesellschaft für Osteuropakunde e.V. und

Internationales Bildungs- und Begegnungswerk, Dortmund

Die Meinungen, die in den Belarus-Analysen geäußert werden, geben ausschließlich die Auffassung der Autoren wieder.

Abdruck und sonstige publizistische Nutzung sind nach Rücksprache mit der Redaktion gestattet.

Redaktion: Astrid Sahm (verantwortlich), Olga Dryndova

Satz: Matthias Neumann

Belarus-Analysen-Layout: Cengiz Kibaroglu, Matthias Neumann und Michael Clemens

Alle Ausgaben der Belarus-Analysen sind mit Themen- und Autorenindex archiviert unter < www.laender-analysen.de> ISSN 2192-1350 @ 2014 by Forschungsstelle Osteuropa, Bremen

Forschungsstelle Osteuropa • Publikationsreferat • Klagenfurter Str. 3 • 28359 Bremen • Telefon: +49 421-218-69600 • Telefax: + 49 421-218-69607 e-mail: publikationsreferat@osteuropa.uni-bremen.de•Internet-Adresse: <http://www.laender-analysen.de/belarus/> 


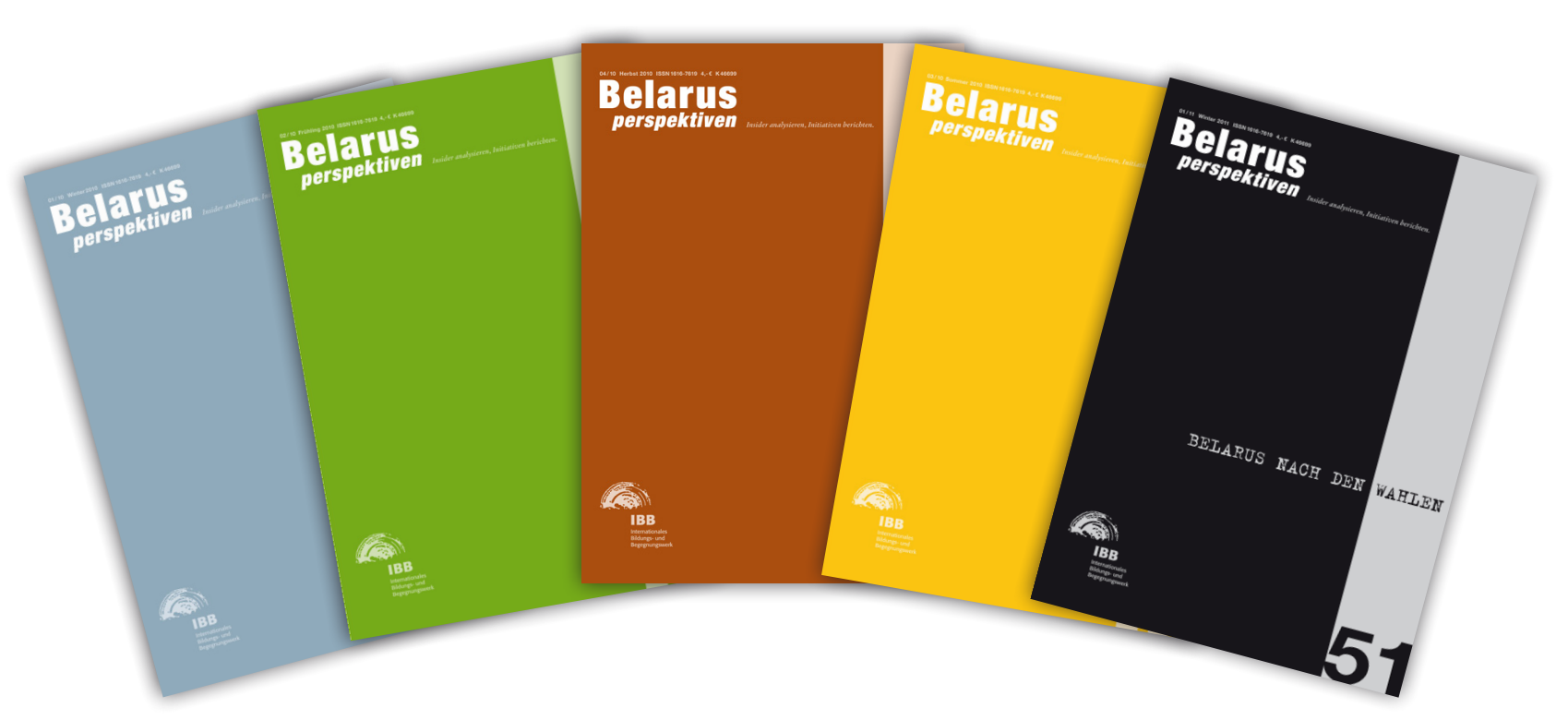

\section{Belarus \\ Perspektiven}

- Seit mehr als zehn Jahren berichten die Belarus Perspektiven aus einem fast unbekannten Land. Uns lesen Initiativen und Politiker, Journalisten und Unternehmer - all jene, die in Belarus etwas bewegen wollen.

- Durch unsere kritischen Berichte und Analysen sind unsere Leser nicht nur auf dem neuesten Stand sie erfahren auch, was hinter den Kulissen geschieht.

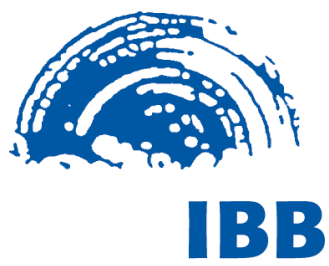

Internationales Bildungs- und Begegnungswerk
- Autoren der Belarus Perspektiven sind namhafte belarussische Journalisten, unabhängige Medienvertreter, Wirtschaftsexperten und zivilgesellschaftliche Akteure.

- Die Zeitschrift erscheint vierteljährlich und kostet inklusive Versand $15,-€$ im Jahresabonnement.

- Zu beziehen sind die Belarus Perspektiven beim IBB in Dortmund bzw. online unter <http://www.ibb-d.de/publikation-anfordernhtml.html>

- Ältere Ausgaben der Belarus Perspektiven finden Sie im Archiv als pdf-Datei zum Download, <http://www.ibb-d.de/bp-archiv.html> 


\title{
Kostenlose E-Mail-Dienste der Forschungsstelle Osteuropa und ihrer $\square ; \square$ Partner auf www.laender-analysen.de
}

Die Länder-Analysen bieten regelmäßig kompetente Einschätzungen aktueller politischer, wirtschaftlicher, sozialer und kultureller Entwicklungen in Ostmitteleuropa und der GUS. Sie machen das Wissen, über das die wissenschaftliche Forschung in reichem Maße verfügt, für Politik, Wirtschaft, Medien und die interessierte Öffentlichkeit verfügbar. Autoren sind internationale Fachwissenschaftler und Experten.

Die einzelnen Länder-Analysen werden von der Forschungsstelle Osteuropa an der Universität Bremen und der Deutschen Gesellschaft für Osteuropakunde jeweils mit unterschiedlichen Partnern und Sponsoren herausgegeben.

Die Länder-Analysen bieten regelmäßig Kurzanalysen zu aktuellen Themen, ergänzt um Grafiken und Tabellen sowie Dokumentationen. Zusätzlich gibt es eine Chronik aktueller Ereignisse.

\author{
Belarus-Analysen \\ Erscheinungsweise: zweimonatlich \\ Abonnement unter: <http://www.laender-analysen.de/belarus/>
}

\section{Caucasus Analytical Digest}

In englischer Sprache. Erscheinungsweise: monatlich

Abonnement unter: $<$ http://www.css.ethz.ch/publications/newsletter_CAD_EN>

\section{Polen-Analysen}

Erscheinungsweise: zweimal monatlich

Abonnement unter: <http://www.deutsches-polen-institut.de/Newsletter/subscribe.php>

\section{Russland-Analysen}

Erscheinungsweise: zweiwöchentlich

Abonnement unter: <http://www.laender-analysen.de/russland/>

\section{Russian Analytical Digest}

In englischer Sprache. Erscheinungsweise: zweimal monatlich

Abonnement unter: $<$ http://www.css.ethz.ch/publications/newsletter_RAD_EN>

\section{Ukraine-Analysen}

Erscheinungsweise: zweimal monatlich

Abonnement unter: <http://www.laender-analysen.de/ukraine/>

\section{Zentralasien-Analysen}

Erscheinungsweise: monatlich

Abonnement unter: <zentralasien-analysen@dgo-online.org>

\section{Bibliographische Dienste}

Die Bibliographien informieren über englisch- und deutschsprachige Neuerscheinungen zu Belarus, Russland, Ukraine sowie zu den zentralasiatischen und kaukasischen Staaten. Erfasst werden jeweils die Themenbereiche Politik, Außenpolitik, Wirtschaft und Soziales.

Erscheinungsweise: viermal jährlich

Abonnement unter: Belarus: <http://www.laender-analysen.de/bibliographies/belarus.php>; Russland: <http://www. laender-analysen.de/bibliographies/russia.php>; Ukraine: <http://www.laender-analysen.de/bibliographies/ukraine. php>; zentalasiatische und kaukasische Staaten: <http://www.laender-analysen.de/bibliographies/caucasus_ca.php>

\section{Twitter}

<https://twitter.com/laenderanalysen> 